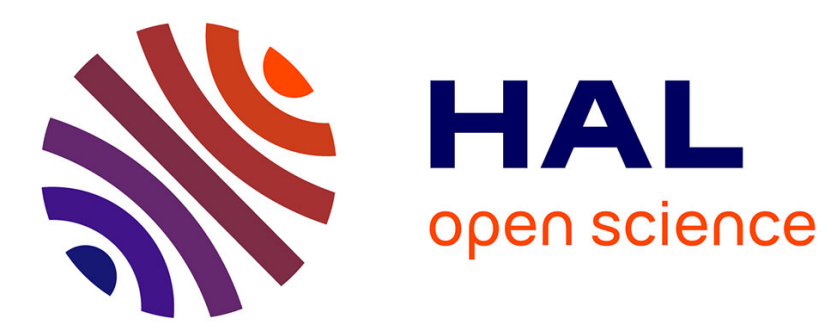

\title{
Optimal Shape Design of Inductor Coils for Surface Hardening
}

\author{
Dietmar Hömberg, Jan Sokolowski
}

\section{To cite this version:}

Dietmar Hömberg, Jan Sokolowski. Optimal Shape Design of Inductor Coils for Surface Hardening. [Research Report] RR-3782, INRIA. 1999, pp.26. inria-00072879

\section{HAL Id: inria-00072879 \\ https://hal.inria.fr/inria-00072879}

Submitted on 24 May 2006

HAL is a multi-disciplinary open access archive for the deposit and dissemination of scientific research documents, whether they are published or not. The documents may come from teaching and research institutions in France or abroad, or from public or private research centers.
L'archive ouverte pluridisciplinaire HAL, est destinée au dépôt et à la diffusion de documents scientifiques de niveau recherche, publiés ou non, émanant des établissements d'enseignement et de recherche français ou étrangers, des laboratoires publics ou privés. 


\section{Optimal shape design of inductor coils for surface hardening}

Dietmar Hömberg, Jan Sokołowski

No 3782

Octobre 1999

THÈME 4 



\title{
Optimal shape design of inductor coils for surface hardening
}

\author{
Dietmar Hömberg*, Jan Sokołowski ${ }^{\dagger}$ \\ Thème 4 - Simulation et optimisation \\ de systèmes complexes \\ Projet Numath
}

Rapport de recherche $\mathrm{n}^{\circ} 3782$ - Octobre $1999-26$ pages

Résumé : A shape optimization problem is considered related to the design of induction hardening facilities. The mathematical model consists of a vector potential formulation for Maxwell's equations coupled with the energy balance and an ODE to describe the solid-solid phase transition in steel during heating. Depending on the shape of the coil we control the volume fraction of the high temperature phase. The coil is modeled as a tube and is defined by a unit-speed curve. The shape optimization problem is formulated over the set of admissible curves. The existence of an optimal control is proved. To obtain the form of the shape gradient of the cost functional, the material derivative method is applied. Finally, the first order necessary optimality conditions are estabished for an optimal tube.

Mots-clé : shape optimization, surface hardening, heat equation, Maxwell's equations, optimality conditions

* Weierstraß-Institut für Angewandte Analysis und Stochastik, Mohrenstraße 39, D - 10117 Berlin, Germany; email:hoemberg@wias-berlin.de

† IECN/Inria-Lorraine, BP 239, 54506 Vandoeuvre lès Nancy, France; e-mail: sokolows@iecn.u-nancy.fr Unité de recherche INRIA Lorraine

Technopôle de Nancy-Brabois, Campus scientifique, 615 rue de Jardin Botanique, BP 101, 54600 VILLERS LÈS NANCY (France)

Téléphone : 0383593030 - International : +33 3383593030

Télécopie : 0383278319 - International : +33 383278319 


\title{
Un problème d'optimisation de forme relatif à la modélisation d'endurcissement par induction
}

\begin{abstract}
Nous considérons un problème d'optimisation de forme relatif à la modélisation d'endurcissement par induction. Le modèle mathématique consiste en une formulation potentielle vectorielle pour les équations de Maxwell couplée au bilan d'énergie et à une EDO afin de décrire la phase de transition solide-solide dans l'acier pendant le chauffage. En agissant sur la forme de l'anneau, nous contrôlons la fraction de volume de la phase à haute température. L'anneau est modélisé par un tube et est défini par une courbe de vitesse unité. Le problème d'optimisation de forme est formulé pour l'ensemble des courbes admissibles. Nous prouvons l'existence d'un contrôle optimal. On utilise la méthode de la dérivée matérielle afin d'obtenir le gradient de forme de la fonctionnelle de coût. Enfin, on établit, dans le cas d'un tube optimal, les conditions nécessaires d'optimalité du premier ordre.
\end{abstract}

Key-words: optimisation de forme, équation non-linéaire de la chaleur, équations de Maxwell, conditions nécessaires d'optimalité 


\section{Introduction}

We investigate the problem of finding the optimal design for an inductor coil in induction hardening machines. The mode of operation of these machines relies on the transformer principle. A given current density in the inductor coil induces eddy currents inside the workpiece. Because of the Joule effect these eddy currents lead to an increase in temperature in the boundary layers of the workpiece. Then the current is switched off and the workpiece is quenched by spray-water cooling. The solid-solid phase transitions during this heat treatment lead to the desired hardening effect.

For an induction hardening machine there are generally speaking two control parameters. One is the frequency of the AC-current applied. This is predefined by choosing a particular machine. Thereby, also the greatest possibe hardening depth is fixed, since it depends on frequency through the skin effect.

The second control parameter is the shape of the inductor coil. These coils are made individually for the specific workpiece from long copper tubes of quadratic or circular cross section. The design of decent coils for specific hardening purposes up to now mostly depends on experience.

However, there is a growing demand in industry for a more precise process control, mainly for two reasons. One is the general goal of weight reduction especially in automotive industry, leading to components made of thinner and thinner steel sheets. Surface hardening of these sheets is a very delicate task, since one must be careful not to harden the complete sheet, which would lead to undesirable fatigue effects. The second one is the tendency for using high quality steels with only small carbon content, which again demands for a very precise process control, now for metallurgical reasons, since the hardenability of a steel is directly related to its carbon content.

There are already numerous papers on modeling and simulation of induction heating machines, e.g. [6], [8], [10], [14]; results on the mathematical analysis can be found in [2]. Models for phase transitions in steel have been investigated in [10], [12] and [18]. Optimal control problems in the case of laser surface hardening have been considered in [1], [13], and for a 2D induction heating problen in [3].

In this paper for the first time a control problem for the 3D induction heating process including phase transitions is investigated. In Sec. 2 we derive the model, consisting of a vector potential formulation of Maxwell's equations, the balance of internal energy and an ODE to describe the phase transition during heating, and prove its well-posedness. In Sec. 3 we formulate the shape design problem for the inductor coil modeled as a tube with circular cross section. In Sec. 4, we prove the existence of an optimal design and finally in the last section first order necessary optimality conditions are derived.

\section{The state equations}

\subsection{The vector potential formulation of Maxwell's equations}

We consider the following slightly idealized geometric setting (cf. fig. 1). Let $D \subset \mathbb{R}^{3}$ with sufficiently smooth boundary and $\Omega \subset D$ be the coil. Its boundary $\partial \Omega$ is dissected into two parts. In $\Gamma_{1}$ the normal component of the current density will be prescribed, this is where in reality the coil is connected to the primary circuit of the hardening machine. $\Sigma$ is the workpiece to be hardened and $G:=\Omega \cup \Sigma$ the set of conductors. Moreover, we define $Q=\Sigma \times(0, T)$.

In eddy current problems we can neglect displacement currents, hence we consider the following set of Maxwell equations:

$$
\begin{aligned}
\operatorname{curl} H & =J, \\
\operatorname{curl} E & =-B_{t} \\
\operatorname{div} B & =0
\end{aligned}
$$




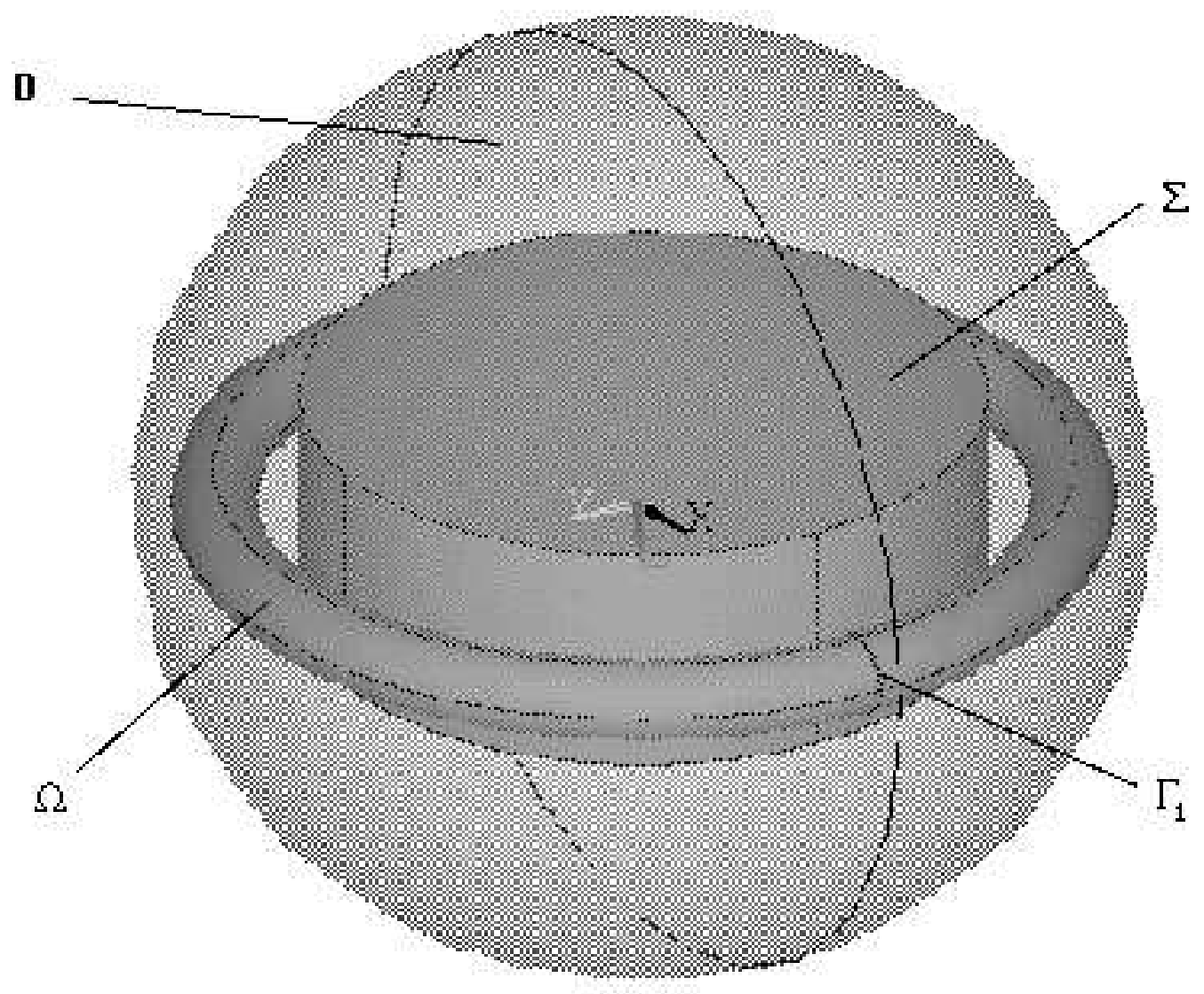

FIG. 1 - The setting

Here, $E$ is the electric field, $B$ the magnetic induction, $H$ the magnetic field and $J$ the current density. In addition we consider the following linear constitutive relations

$$
\begin{aligned}
& J=\sigma E, \quad \text { in } D, \\
& B=\mu H, \quad \text { in } D,
\end{aligned}
$$

with the magnetic permeability $\mu$ and the electric conductivity $\sigma$. We assume zero current density outside conductors, i.e.

$$
\sigma(x)= \begin{cases}\sigma_{0}>0, & \text { in } \bar{G}, \\ 0, & \text { in } D \backslash \bar{G},\end{cases}
$$

The magnetic permeability takes different values in the coil (usually made of copper), in the workpiece and in the surrounding air. Hence, we assume

$$
\mu(x)= \begin{cases}\mu_{1}, & \text { in } \bar{\Omega} \\ \mu_{2}, & \text { in } \bar{\Sigma} \\ \mu_{3}, & \text { in } D \backslash(\bar{\Omega} \cup \bar{\Sigma}) .\end{cases}
$$

Using $(2.1 \mathrm{a}, \mathrm{b})$ one now introduces the magnetic vector potential $A$ and the scalar potential $\phi$ such that

$$
\begin{aligned}
& B=\operatorname{curl} A, \\
& E=-A_{t}-\operatorname{grad} \phi .
\end{aligned}
$$


Then, Maxwell's equations $(2.1 \mathrm{a}-\mathrm{c})$ can be rewritten in the following way:

$$
\sigma A_{t}+\operatorname{curl}\left(\frac{1}{\mu} \operatorname{curl} A\right)+\sigma \operatorname{grad} \phi=0, \quad \text { in } D .
$$

The scalar potential $\phi$ is determined by the continuity equation $\operatorname{div} J=0$, i.e.

$$
-\operatorname{div}\left(\sigma \operatorname{grad} \phi+\sigma A_{t}\right)=0, \quad \text { in } G .
$$

Since $B$ is not uniquely defined by (2.3), we impose the Coulomb gauge

$$
\operatorname{div} A=0 .
$$

Then $(2.5 \mathrm{~b})$ can be reduced to the Laplace equation

$$
-\Delta \phi=0, \quad \text { in } G
$$

with boundary condition

$$
-\sigma_{0} \frac{\partial \phi}{\partial n}= \begin{cases}j_{g}, & \text { in } \Gamma_{1}, \\ 0, & \text { in } \partial \Sigma \cup \Gamma_{2},\end{cases}
$$

with $\Gamma_{2}=\partial \Omega \backslash \Gamma_{1}$.

The system $(2.7 \mathrm{a}, \mathrm{b})$ is a linear elliptic problem, which can be solved separately in $\Omega$ and $\Sigma$. At the workpiece boundary $\partial \Sigma$, we have homogenous boundary conditions, i.e. the solution is constant in $\Sigma$. Since only the gradient enters in (2.5a), we restrict the domain of $\phi$ to the coil $\Omega$.

Assuming that the tangential component of $A$ vanishes on $\partial D$, i.e.

$$
n \times A=0,
$$

we introduce the spaces

$$
\begin{aligned}
H(\operatorname{curl}, D) & =\left\{v \in \mathbf{L}^{2}(D) ; \operatorname{curl} v \in \mathbf{L}^{2}(D)\right\} \\
H_{0}(\operatorname{curl}, D) & =\left\{v \in H(\operatorname{curl}, D) ; n \times\left. v\right|_{\partial D}=0\right\} \\
H(\operatorname{div}, D) & =\left\{v \in \mathbf{L}^{2}(D) ; \operatorname{div} v \in \mathbf{L}^{2}(D)\right\}
\end{aligned}
$$

where $\mathbf{L}$ denotes the vector-valued counterpart $\mathbf{L}=[L]^{3}$ for any real-valued Sobolev space $L$. Then, there holds (cf. [7]):

$$
H_{0}(\operatorname{curl}, D) \cap H(\operatorname{div}, D)=\left\{v \in \mathbf{H}^{1}(D) ; n \times\left. v\right|_{\partial D}=0\right\} .
$$

Finally, we introduce the Hilbert space

$$
\mathbf{X}=\left\{v \in H_{0}(\operatorname{curl}, D) ; \operatorname{div} v=0\right\},
$$

which is a closed subspace of $\mathbf{H}^{1}(D)$, equipped with the norm

$$
\|v\|_{\mathbf{X}}=\|\operatorname{curl} v\|_{\mathbf{L}^{2}(D)}
$$

and recall the Green's formula

$$
<n \times f, g>=\int_{D} \operatorname{curl} f \cdot g d x-\int_{D} f \cdot \operatorname{curl} g d x,
$$

for all $f \in H(\operatorname{curl}, D)$ and $g \in \mathbf{H}^{1}(D)$, where $<., .>$ denotes the duality pairing between $\mathbf{H}^{-1 / 2}(\partial D)$ and $\mathbf{H}^{1 / 2}(\partial D)$.

We assume

(H1) $j_{g} \in H^{1}\left(0, T ; H^{1}\left(\Gamma_{1}\right)\right)$, such that $\int_{\Gamma_{1}} j_{g} d x=0$, and $\int_{\Gamma_{1}} j_{g, t} d x=0$,
RR n ${ }^{\circ} 3782$ 
(H2) $A_{0} \in \mathbf{X}$.

Using (2.8) and (2.9) we obtain the following weak formulation of $(2.5 \mathrm{a}),(2.7 \mathrm{a}, \mathrm{b})$ :

Find $(A, \phi)$, such that $A(0)=A_{0}$,

$$
\begin{aligned}
\sigma_{0} \int_{G} A_{t} \cdot v d x+\int_{D} \frac{1}{\mu} \operatorname{curl} A \cdot \operatorname{curl} v d x+\sigma_{0} \int_{\Omega} \nabla \phi \cdot v d x & =0 \\
\sigma_{0} \int_{\Omega} \nabla \phi \cdot \nabla u d x+\int_{\Gamma_{1}} j_{g} u d x & =0
\end{aligned}
$$

for all $v \in \mathbf{X}$ and $u \in H^{1}(\Omega)$.

In view of (H1), we obtain easily:

Lemma 2.1 Assume (H1), then (2.11) has a solution. Moreover, $\nabla \phi$ is uniquely defined and satisfies

$$
\|\nabla \phi\|_{H^{1}\left(0, T ; \mathbf{L}^{2}(\Omega)\right)} \leq C,
$$

where $C$ only depends on $T$ and $j_{g}$.

We assume further

(H3) There exists $y_{0} \in \mathbf{X}$ such that

$$
\sigma_{0} \int_{G} y_{0} \cdot v d x+\int_{D} \frac{1}{\mu} \operatorname{curl} A_{0} \cdot \operatorname{curl} v d x+\sigma_{0} \int_{\Omega} \nabla \phi \cdot \nabla v d x=0,
$$

for all $v \in \mathbf{X}$, where $\phi$ is the unique solution to (2.11) (cf. [16]).

Then, we have

Lemma 2.2 Assume (H1)-(H3) and let $\phi$ be the unique solution to (2.11). Then, (2.10) has a unique solution $A \in L^{\infty}(0, T ; \mathbf{X})$. Moreover, there exists a constant $C>0$ such that

$$
\left\|A_{t}\right\|_{L^{4}\left(0, T ; L^{4}(G)\right)} \leq C .
$$

Proof:

Uniqueness follows from standard arguments. To prove existence, we fix $M \in \mathbb{N}$ and define $h=$ $T / M, A^{0}=A_{0}$, and

$$
\phi^{m}(x)=\frac{1}{h} \int_{(m-1) h}^{m h} \phi(x, t) d t \quad \text { for } m \in\{1, \ldots M\} .
$$

Then, we consider the time-discrete version of (2.10):

$$
\sigma_{0} \int_{G} \delta_{h} A^{m} \cdot v d x+\int_{D} \frac{1}{\mu} \operatorname{curl} A^{m} \cdot \operatorname{curl} v d x+\sigma_{0} \int_{\Omega} \nabla \phi^{m} \cdot v d v=0 \text { for all } v \in \mathbf{X}
$$

for $m \in\{1, \ldots, M\}$, with $\delta_{h} A^{m}=\frac{1}{h}\left(A^{m}-A^{m-1}\right)$.

Owing to (H2), the first two terms in (2.12) define a coercive bilinear form on $\mathbf{X}$, hence applying the Lax-Milgram lemma, inductively we obtain a unique solution $A^{m}$ to $(2.12)$ for $m \in\{1, \ldots, M\}$. Next, we insert $v=A^{m}-A^{m-1}$ into (2.12) and sum up for $m=1, \ldots k$ :

$$
\begin{aligned}
\sigma_{0} \sum_{m=1}^{k} h \int_{G}\left|\delta_{h} A^{m}\right|^{2} d x+\sum_{m=1}^{k} \int_{G} \frac{1}{\mu} \operatorname{curl} A^{m} \cdot\left(\operatorname{curl} A^{m}-\operatorname{curl} A^{m-1}\right) d x \\
+\sigma_{0} \sum_{m=1}^{k} \int_{\Omega} \nabla \phi^{m} \cdot\left(A^{m}-A^{m-1}\right) d x=0 .
\end{aligned}
$$


Using the inequalities of Young and Hölder, we obtain

$$
\begin{aligned}
& \sigma_{0} \sum_{m=1}^{k} \int_{\Omega} \nabla \phi^{m} \cdot\left(A^{m}-A^{m-1}\right) d x \\
& \quad \leq \frac{\sigma_{0}}{2} \sum_{m=1}^{k} h \int_{\Omega}\left|\nabla \phi^{m}\right|^{2} d x+\frac{\sigma_{0}}{2} \sum_{m=1}^{k} h \int_{\Omega}\left|\delta_{h} A^{m}\right|^{2} d x .
\end{aligned}
$$

Routine calculations show

$$
\begin{aligned}
& \sum_{m=1}^{k} \int_{G} \frac{1}{\mu} \operatorname{curl} A^{m} \cdot\left(\operatorname{curl} A^{m}-\operatorname{curl} A^{m-1}\right) d x \\
& \quad=\sum_{m=1}^{k} \int_{G} \frac{1}{\mu}\left|\operatorname{curl} A^{m}\right|^{2} d x-\sum_{m=1}^{k} \int_{G} \frac{1}{\mu} \operatorname{curl} A^{m} \cdot \operatorname{curl} A^{m-1} d x \\
& \quad=\sum_{m=1}^{k} \int_{G} \frac{1}{2 \mu}\left|\operatorname{curl} A^{m}-\operatorname{curl} A^{m-1}\right|^{2} d x+\int_{G} \frac{1}{2 \mu}\left|\operatorname{curl} A^{k}\right|^{2} d x-\int_{G} \frac{1}{2 \mu}\left|\operatorname{curl} A^{0}\right|^{2} d x .
\end{aligned}
$$

Hence, using Lemma 2.1, we obtain

$$
\begin{aligned}
\frac{\sigma_{0}}{2} \sum_{m=1}^{M} h\left\|\delta_{h} A^{m}\right\|_{\mathbf{L}^{2}(G)}^{2}+\frac{1}{2 \mu_{1}} \max _{1 \leq k \leq M}\left\|\operatorname{curl} A^{k}\right\|_{\mathbf{L}^{2}(D)}^{2}+ \\
\quad+\frac{1}{2 \mu_{1}} \sum_{m=1}^{M}\left\|\operatorname{curl} A^{m}-\operatorname{curl} A^{m-1}\right\|_{\mathbf{L}^{2}(D)}^{2} \leq c_{1},
\end{aligned}
$$

with a constant $c_{1}$ independent of $M$.

To obtain higher regularity, we take the difference of (2.12) for $m$ and $m-1$ divided by $h$,

$$
\sigma_{0} \int_{G} \delta_{h}^{2} A^{m} \cdot v d x+\int_{D} \frac{1}{\mu} \operatorname{curl} \delta_{h} A^{m} \cdot \operatorname{curl} v d x+\sigma_{0} \int_{\Omega} \nabla \delta_{h} \phi^{m} \cdot v d x=0 \text { for all } v \in \mathbf{X}
$$

with $\delta_{h}^{2} A^{m}=\frac{1}{h}\left(\delta_{h} A^{m}-\delta_{h} A^{m-1}\right)$. In view of (H3), we define $\delta_{h} A^{0}:=y_{0}$. Then, the previous equation is valid for $m \in\{1, \ldots, M\}$.

Putting $v=\delta_{h} A^{m}-\delta_{h} A^{m-1}$, in the same manner as above we get

$$
\begin{aligned}
\frac{\sigma_{0}}{2} \sum_{m=1}^{M} h\left\|\delta_{h}^{2} A^{m}\right\|_{\mathbf{L}^{2}(G)}^{2}+\frac{1}{2 \mu_{1}} \max _{1 \leq k \leq M}\left\|\operatorname{curl} \delta_{h} A^{k}\right\|_{\mathbf{L}^{2}(D)}^{2}+ \\
+\frac{1}{2 \mu_{1}} \sum_{m=1}^{M}\left\|\operatorname{curl} \delta_{h} A^{m}-\operatorname{curl} \delta_{h} A^{m-1}\right\|_{\mathbf{L}^{2}(D)}^{2} \leq c_{2},
\end{aligned}
$$

with a constant $c_{2}$ independent of $M$.

Now, we define approximating functions

$$
\begin{array}{lll}
A_{h}(t)=A^{m}+\frac{t-m h}{h}\left(A^{m}-A^{m-1}\right) & \text { for } t \in[(m-1) h, m h], \\
\hat{A}_{h}=A^{m}, & \text { for } t \in((m-1) h, m h] .
\end{array}
$$

In view of (2.13), we have

$\mathrm{RR} \mathrm{n}^{\circ} 3782$

$$
\begin{aligned}
\left\|A_{h}\right\|_{H^{1}\left(0, T ; \mathbf{L}^{2}(G)\right) \cap L^{\infty}(0, T ; \mathbf{X})} & \leq c_{3} \\
\left\|\hat{A}_{h}\right\|_{L^{\infty}(0, T ; \mathbf{X})} & \leq c_{4}
\end{aligned}
$$


and

$$
\left\|A_{h}-\hat{A}_{h}\right\|_{L^{2}(0, T ; \mathbf{X})}^{2} \leq \frac{h}{3} \sum_{m=1}^{M} \int_{D}\left|\operatorname{curl} A^{m}-\operatorname{curl} A^{m-1}\right|^{2} d x \longrightarrow 0,
$$

for $h \rightarrow 0$. Thus, we have the convergences

$$
\begin{array}{ll}
A_{h} \longrightarrow A, & \text { weakly-star in } L^{\infty}(0, T ; \mathbf{X}) \cap H^{1}\left(0, T ; \mathbf{L}^{2}(G)\right), \\
\hat{A}_{h} \longrightarrow \hat{A}, & \text { weakly-star in } L^{\infty}(0, T ; \mathbf{X}) .
\end{array}
$$

Moreover,

$$
A=\hat{A} \quad \text { a.e. in } D \times(0, T)
$$

and $A$ is a solution to $(2.10)$.

Now, let $y_{m}=\delta_{h} A^{m}$ and

$$
\begin{aligned}
& A_{h}^{(1)}(t)=y^{m}+\frac{t-m h}{h}\left(y^{m}-y^{m-1}\right) \quad \text { for } t \in[(m-1) h, m h], \\
& {\hat{A_{h}}}^{(1)}=y^{m}, \quad \text { for } t \in((m-1) h, m h] \text {. }
\end{aligned}
$$

As before, we get

$$
\begin{aligned}
& A_{h}^{(1)} \longrightarrow y, \quad \text { weakly-star in } L^{\infty}(0, T ; \mathbf{X}) \cap H^{1}\left(0, T ; \mathbf{L}^{2}(G)\right), \\
& \text { strongly in } L^{2}\left(0, T ; \mathbf{L}^{2}(G)\right) \\
& \hat{A}_{h}^{(1)} \longrightarrow \hat{y}, \quad \text { weakly-star in } L^{\infty}(0, T ; \mathbf{X}),
\end{aligned}
$$

and $y=\hat{y}$ a.e. in $D \times(0, T)$. On the other hand, we have

$$
\hat{A}_{h}^{(1)}=A_{h, t} \longrightarrow A_{t}, \quad \text { weakly in } L^{2}\left(0, T ; \mathbf{L}^{2}(G)\right) .
$$

Hence, we conclude

$$
y=A_{t} \quad \text { a.e. in } G \times(0, T) .
$$

Using Sobolev embedding theorem we finally obtain

$$
\left\|A_{t}\right\|_{L^{4}\left(0, T ; \mathbf{L}^{4}(G)\right)} \leq\|y\|_{L^{\infty}(0, T ; \mathbf{X})} \leq c_{4} .
$$

\subsection{Energy balance and the formation of austenite}

A good measure for the hardness penetration depth in the workpiece is the formation of austenite during heating, which can be described by the following initial-value problem derived by Leblond \& Deveaux [15] (for details, we refer to [10]):

$$
\begin{aligned}
z(0) & =0 \\
z_{t}(t) & =\frac{1}{a(\theta)}[b(\theta)-z]^{+}
\end{aligned}
$$

where $z$ is the volume fraction of austenite and $\theta$ the temperature. To avoid technical difficulties, we assume for the positive part function [.] ${ }^{+}$and the temperature dependent coefficients $a, b$ :

(H4) $[x]^{+}=x \cdot \mathcal{H}(x)$, where $\mathcal{H}$ is a regularized, smooth Heaviside function,

$(\mathrm{H} 5) b \in C^{1,1}(\mathbb{R}), b(x) \in[0,1]$ for all $x \in \mathbb{R}$, 
(H6) $a \in C^{1,1}(\mathbb{R}), m \leq a(x) \leq M$ for all $x \in \mathbb{R}$, and constants $0<m<M$.

We consider the following semi-linear energy balance equation:

$$
\begin{aligned}
\rho c_{p} \theta_{t}-\operatorname{div}(k \operatorname{grad} \theta) & =-\rho L z_{t}+\sigma_{0}\left|A_{t}\right|^{2}, \quad \text { in } \Sigma \times(0, T) \\
\frac{\partial \theta}{\partial n} & =0, \quad \text { in } \partial \Sigma \times(0, T) \\
\theta(., 0) & =\theta_{0} \quad \text { in } \Sigma .
\end{aligned}
$$

$\rho, c_{p}, k, L$ are density, specific heat at constant pressure, heat conductivity and latent heat, assumed to be constant. The first term on the right-hand side of $(2.17 \mathrm{a})$ measures the latent heat inside the workpiece $\Sigma$, which is consumed during the formation of austenite. The second one describes the Joule heating $\sigma_{0}|E|^{2}$, cf. (2.4). Note that $\nabla \phi \equiv 0$ in $\Sigma$.

Using the results of [12], we can easily prove

Lemma 2.3 Assume (H4)-(H6) and let $\theta_{0} \in H^{1}(\Sigma)$. Then (2.16a,b), (2.17a-c) has a unique solution $(z, \theta) \in W^{1, \infty}\left(0, T ; L^{\infty}(\Sigma)\right) \times H^{2,1}(Q)$.

Here, we have used the abbreviation $Q=\Sigma \times(0, T)$.

\section{The shape design problem}

The technological aim is to obtain a certain, possibly uniform penetration depth of austenite inside the workpiece. The most important control parameter to achieve this goal is the shape of the coil $\Omega$. Hence, we consider the following cost functional:

$$
J(\Omega)=\int_{\Sigma}(z(x, T)-\bar{z}(x))^{2} d x,
$$

where $\bar{z}$ is a given distribution of austenite.

Note that the cost functional depends on $\Omega$ only implicitely, through the solution to the Maxwell equation (2.10).

Inductor coils are manufactured from copper tubes with approximately quadratic cross-section. For convenience, we will consider tubes with circular cross-section. These tubes can easily be generated from curves in the following way (cf. Gray, [11]).

Let $\gamma:[0, l] \longrightarrow \mathbb{R}^{3}$ be a unit speed curve, i.e.

$$
|T(s)|=1, \quad s \in[0, l],
$$

where $T(s)=\gamma^{\prime}(s)$ is the unit tangential vector. Since the arc-length of a unit-speed curve is given by

$$
\int_{0}^{t}\left|\gamma^{\prime}(s)\right| d s=t
$$

unit-speed curves are said to be parametrized by arc length. If the curvature $\kappa(s)=\left|\gamma^{\prime \prime}(s)\right|$ does not vanish, we can define the unit normal and binormal vector fields $N$ and $B$, respectively, by

$$
\begin{aligned}
N(s) & =\frac{1}{\kappa(s)} T^{\prime}(s), \\
B(s) & =T(s) \times N(s) .
\end{aligned}
$$

If $\kappa(s)=0$, one can easily choose two vectors $N, B$ to form an orthogonal system with $T(s)$.

$\mathrm{RR} \mathrm{n}^{\circ} 3782$ 
Then, the tube with circular cross-section $R>0$ corresponding to $\gamma$ is given by

$$
\Omega=\Omega(\gamma)=\left\{\omega_{\gamma}(s, r, \vartheta) \mid 0 \leq s \leq l, 0 \leq r \leq R, 0 \leq \vartheta \leq 2 \pi\right\}
$$

with

$$
\omega_{\gamma}(s, r, \vartheta)=\gamma(s)+r \cos \vartheta N(s)+r \sin \vartheta B(s) .
$$

The faces of the tube, i.e. the parts, where the inductor is connected to the hardening machine (cf. $(2.7 \mathrm{~b}))$ are defined by

$$
\Gamma_{1}=\left\{\omega_{\gamma}(0, r, \vartheta) \mid 0 \leq r \leq R, 0 \leq \vartheta \leq 2 \pi\right\} \cup\left\{\omega_{\gamma}(l, r, \vartheta) \mid 0 \leq r \leq R, 0 \leq \vartheta \leq 2 \pi\right\} .
$$

The lateral boundary is parametrized by

$$
\Gamma_{2}=\left\{\omega_{\gamma}(s, R, \vartheta) \mid 0 \leq s \leq l, 0 \leq \vartheta \leq 2 \pi\right\}
$$

Therefore, $\partial \Omega=\Gamma_{1} \cup \Gamma_{2}$.

Even simply connected curves may generate tubes with intersecting parts. For obvious technical reasons this case has to be excluded. To this end we introduce the notion of reach (cf. Federer [9]).

Definition 3.1 (Federer, 1959)

Let $\boldsymbol{\Gamma}_{\gamma}=\{\gamma(s), s \in[0, l]\}$ be the trace of $\gamma$. We call $\operatorname{Unp}\left(\boldsymbol{\Gamma}_{\gamma}\right)$ the set of all points $x \in \mathbb{R}^{3}$, for which there exists a unique projection onto $\boldsymbol{\Gamma}_{\gamma}$. For $y \in \boldsymbol{\Gamma}_{\gamma}$ we define

$$
\begin{aligned}
\operatorname{reach}\left(\boldsymbol{\Gamma}_{\gamma}, y\right) & =\sup \left\{r,\left\{x \in \mathbb{R}^{3},|x-y|<r\right\} \subset \operatorname{Unp}\left(\boldsymbol{\Gamma}_{\gamma}\right)\right\}, \quad \text { and } \\
\operatorname{reach}\left(\boldsymbol{\Gamma}_{\gamma}\right) & =\inf \left\{\operatorname{reach}\left(\boldsymbol{\Gamma}_{\gamma}, y\right), y \in \boldsymbol{\Gamma}\right\} .
\end{aligned}
$$

In other words, reach of a subset $B \subset \mathbb{R}^{n}$ is the largest $\varepsilon$ such that for all $x$ in an $\varepsilon$-surrounding of $B$, there exists a unique projection onto $B$. If $B$ is convex, then reach $(B)=\infty$. On the other hand, if $B$ is concave with a reentrant corner, e.g. an L-shaped domain, then reach $(B)=0$. In our situation, to avoid too narrow twists of the curve $\gamma$, we demand

$$
\operatorname{reach}\left(\boldsymbol{\Gamma}_{\gamma}\right) \geq R+\delta
$$

where $\delta>0$ is a given positive parameter and $R$ is the tube radius.

Now we can introduce the set of admissible curves

$$
\begin{gathered}
U_{a d}=\left\{\gamma:[0, l] \rightarrow \mathbb{R}^{3} \backslash U_{R+\delta}(\Sigma),\left|\gamma^{\prime}\right|=1 \text { in }[0, l], \text { reach }\left(\boldsymbol{\Gamma}_{\gamma}\right) \geq R+\delta\right. \\
0<L_{1} \leq l \leq L_{2},\|\gamma\|_{H^{4}[0, l]} \leq M, \gamma(0)=\gamma(l)=0, \\
\left.\gamma^{\prime}(0)=\gamma^{\prime}(l), \gamma^{\prime \prime}(0)=\gamma^{\prime \prime}(l), \gamma^{\prime \prime \prime}(0)=\gamma^{\prime \prime \prime}(l)\right\},
\end{gathered}
$$

the corresponding set of admissible domains

$$
\mathcal{U}_{a d}=\left\{\Omega(\gamma), \gamma \in U_{a d}\right\}
$$

and give a precise definition of our control problem, which reads

(CP) Minimize $J(\Omega)$, given by (3.1) subject to

$\Omega \in \mathcal{U}_{a d}$

and the state equations (2.10), (2.11), (2.16), (2.17).

Note that $(\mathrm{CP})$ is a non-convex optimization (control) problem due to the non-convexity of $\mathcal{U}_{a d}$ and of the cost functional.

For the derivation of optimality conditions it will be convenient to introduce perturbations of the admissible curves, hence we introduce the further notation

$$
I(\gamma)=J(\Omega(\gamma))
$$




\section{The existence of an optimal domain $\Omega^{*}$}

\section{Theorem 4.1}

Assume (H1)-(H6), then (CP) admits a solution $\Omega^{*} \in \mathcal{U}_{a d}$.

For the proof we take a minimizing sequence $\left\{\Omega_{n}\right\} \subset \mathcal{U}_{a d}$ for (3.1). We have $\Omega_{n}=\Omega\left(\gamma_{n}\right)$ and $\gamma_{n}:\left[0, l_{n}\right] \rightarrow \mathbb{R}^{n} \backslash U_{\delta}(\Sigma)$. We extend $\gamma_{n}$ by defining

$$
\gamma_{n}(s)=\sum_{k=0}^{3} \gamma_{n}^{(k)}\left(l_{n}\right)\left(s-l_{n}\right)^{k}, \quad \text { for } s \in\left[l_{n}, L_{2}\right] .
$$

Hence $\left\{\gamma_{n}\right\}$ is bounded in $H^{4}\left(0, L_{2}\right)$ and there exists a subsequence (still indicated by $n$ ) satisfying

$$
\gamma_{n} \rightarrow \gamma^{*} \quad \text { in } C^{3}\left(0, L_{2}\right)
$$

Extracting possibly a further subsequence we also have $l_{n} \rightarrow l^{*} \in\left[L_{1}, L_{2}\right]$. Obviously, $\gamma$ is a unit-speed curve satisfying also (3.4). Let $P=0$ be the prescribed endpoint of the curves defined in $\mathcal{U}_{a d}$, then

$$
\left|\gamma_{n}\left(l^{*}\right)-P\right| \leq\left|\gamma_{n}\left(l_{n}\right)-P\right|+\left|\gamma_{n}\left(l^{*}\right)-\gamma_{n}\left(l_{n}\right)\right| \leq c\left|l^{*}-l_{n}\right| \rightarrow 0, \text { for } n \rightarrow \infty .
$$

In the same manner, we obtain $\gamma^{*^{\prime}}(0)=\gamma^{*^{\prime}}\left(l^{*}\right), \gamma^{*^{\prime \prime}}(0)=\gamma^{*^{\prime \prime}}\left(l^{*}\right)$ and $\gamma^{*^{\prime \prime \prime}}(0)=\gamma^{*^{\prime \prime \prime}}\left(l^{*}\right)$, and thus, we have $\Omega^{*}=\Omega\left(\gamma^{*}\right) \in \mathcal{U}_{a d}$.

For an arbitrary curve $\gamma$ the lateral boundary $\Gamma_{2}$ of the corresponding tube is parametrized by the function $\tilde{\omega}_{\gamma}(s, \vartheta)=\omega_{\gamma}(s, R, \vartheta)$ defined in (3.2). The normal and binormal vectors $N(s), B(s)$ depend on the second derivative of $\gamma$, therefore $\tilde{\omega}_{\gamma}(s, \vartheta)$ is a $C^{1}$ function and the lateral boundary $\Gamma_{2}$ is uniformly Lipschitz continuous, with a Lipschitz constant depending on $M$ in the definition of the family $\mathcal{U}_{a d}$. Therefore, for the sequence $\left\{\Omega_{n}\right\}$ corresponding to our subsequence of curves $\left\{\gamma_{n}\right\}$ selected above, we have the following properties:

$$
\begin{aligned}
& D \backslash \overline{\Omega_{n}} \longrightarrow D \backslash \overline{\Omega^{*}} \quad \text { in the sense of Hausdorf metric, } \\
& \chi_{n}=\chi_{\Omega_{n}} \longrightarrow \chi=\chi_{\Omega^{*}} \quad \text { in } L^{p}(D) \text { for all } p<\infty, \\
& \text { for all } K \subset \subset \Omega^{*} \text { there exists } N \text { such that } K \subset \subset \Omega_{n} \text { for all } n>N .
\end{aligned}
$$

We refer the reader to [5] for the compactness results in the class of uniformly Lipschitz domains. Using these properties, in the following Lemmas we are going to pass to the limit for $n \rightarrow \infty$ in the state equations and show the convergence of solutions, which implies $J\left(\Omega_{n}\right) \rightarrow J\left(\Omega^{*}\right)$ and, therefore the existence of an optimal domain $\Omega^{*}=\Omega\left(\gamma^{*}\right)$.

We begin with the equation for the scalar potential (2.11). Denoting

$$
\widetilde{\nabla \phi_{n}}(x)= \begin{cases}\nabla \phi_{n}(x) & , x \in \Omega_{n}, \\ 0 & , x \in D \backslash \Omega_{n} .\end{cases}
$$

we have

$$
\sigma_{0} \int_{D} \chi_{n} \widetilde{\nabla \phi_{n}} \cdot \nabla u d x+\int_{\Gamma_{1}} j_{g} u d x=0, \quad \text { for all } u \in H^{1}(D)
$$

and obtain

Lemma 4.1 There exists a subsequence satisfying

$$
\widetilde{\nabla \phi_{n}} \longrightarrow \widetilde{\nabla \phi} \quad \text { strongly in } H^{1}\left(0, T ; \mathbf{L}^{2}(D)\right) \text {. }
$$

$\mathrm{RR} \mathrm{n}^{\circ} 3782$ 
Proof:

Since $\left\{\widetilde{\nabla \phi_{n}}\right\}$ is bounded in $L^{2}\left(0, T ; \mathbf{L}^{2}(D)\right),(4.3)$ holds weakly in $L^{2}\left(0, T ; \mathbf{L}^{2}(D)\right)$. Moreover, taking $u=\widetilde{\nabla \phi_{n}}$ in $(4.2)$ we have

$$
\int_{D}\left|\widetilde{\nabla \phi_{n}}\right|^{2} d x=-\frac{1}{\sigma_{0}} \int_{\Gamma_{1}} j_{g} \phi_{n} d x \longrightarrow-\frac{1}{\sigma_{0}} \int_{\Gamma_{1}} j_{g} \phi d x=\int_{D}|\widetilde{\nabla \phi}|^{2} d x
$$

and thus, strong convergence in $L^{2}\left(0, T ; \mathbf{L}^{2}(D)\right)$. Differentiating (4.2) formally with respect to $t$ and reasoning as above completes the proof.

Now, we consider the equation for the magnetic vector potential (2.10). We denote

$$
\mu_{n}(x)= \begin{cases}\mu_{1} & , \text { in } \overline{\Omega_{n}}, \\ \mu_{2} & , \text { in } \bar{\Sigma} \\ \mu_{3} & , \text { in } D \backslash\left(\overline{\Omega_{n}} \cup \bar{\Sigma}\right)=D \backslash \overline{G_{n}},\end{cases}
$$

which can be expressed using characteristic functions as

$$
\mu_{n}=\mu_{1} \chi_{n}+\mu_{2} \chi_{\Sigma}+\mu_{3}\left(1-\chi_{n}-\chi_{\Sigma}\right) .
$$

According to (4.1b), we have

$$
\mu_{n} \longrightarrow \mu \text { in } L^{p}(D) \text { for all } p \in[1, \infty)
$$

We rewrite $(2.10)$ as

$$
\sigma_{0} \int_{G_{n}} A_{t}^{n} \cdot v d x+\int_{D} \frac{1}{\mu_{n}} \operatorname{curl} A^{n} \cdot \operatorname{curl} v d x+\sigma_{0} \int_{D} \widetilde{\nabla \phi_{n}} \cdot v d x=0 .
$$

Making a priori estimates analogous to the discrete ones of Lemma 2.2 (cf. (2.14), (2.15)), $G_{n}=\Omega_{n} \cup \Sigma$, we obtain

Lemma 4.2 There exists a subsequence $\left\{A^{n}\right\}$ satisfying

$$
\begin{aligned}
A^{n} \longrightarrow A, & \text { weakly-star in } L^{\infty}(0, T ; \mathbf{X}) \\
\chi_{G_{n}} A_{t}^{n} \longrightarrow \chi_{G} A_{t}, & \text { weakly in } L^{2}\left(0, T ; L^{2}(D)\right), \\
A_{\left.t\right|_{\Sigma}}^{n} \longrightarrow A_{\left.\right|_{\Sigma}}, & \text { strongly in } C\left(0, T ; L^{4}(\Sigma)\right) .
\end{aligned}
$$

The equations for temperature and phase transition $(2.16),(2.17)$ depend only implicitely on the shape of $\Omega_{n}$, namely through $A^{n}$. Standard a priori estimates for this system yield

$$
\|z\|_{W^{1, \infty}\left(0, T ; L^{\infty}(\Sigma)\right)}+\|\theta\|_{H^{2,1}(Q)} \leq c_{1},
$$

with a constant $c_{1}$ independent of $n$. Hence, using Lebesgue's convergence theorem we obtain

Lemma 4.3 There exist subsequences $\left\{\theta_{n}\right\},\left\{z_{n}\right\}$ satisfying

$$
\begin{aligned}
& \theta_{n} \quad \longrightarrow \quad \theta, \quad \text { strongly in } C\left([0, T] ; H^{1}(\Sigma)\right) \text {, } \\
& z_{n} \quad \longrightarrow \quad z, \quad \text { strongly in } C\left([0, T] ; L^{2}(\Sigma)\right) \text {. }
\end{aligned}
$$

In view of Lemmas $4.1-4.3$, we can pass to the limit in the state equations $(2.10),(2.11),(2.16)$, (2.17) and in the cost functional (3.1), which concludes the proof of Theorem 4.1. 


\section{$5 \quad$ Necessary optimality conditions}

\subsection{Introduction and main results}

We propose the following procedure in order to derive the first order optimality conditions.

Let $\Omega=\Omega(\gamma)$ be an admissible domain. First, we investigate $J(\Omega)$ using the speed method [17]. Given an admissible vector field $V$ with sup $V \cap \partial \Omega \neq \emptyset$ and the associated mapping $T_{\tau}(V): \mathbb{R}^{3} \rightarrow \mathbb{R}^{3}$, we show that there exists the limit

$$
d J(\Omega ; V)=\lim _{\tau \rightarrow 0}\left(J\left(\Omega_{\tau}\right)-J(\Omega)\right),
$$

where $\Omega_{\tau}=T_{\tau}(V)(\Omega)$. Furthermore, the Eulerian derivative $d J(\Omega ; V)$ is linear and continuous with respect to to $V$. Therefore, the shape gradient $g_{\partial \Omega}$ is supported on $\partial \Omega$ and

$$
d J(\Omega ; V)=<g_{\partial \Omega}, V \cdot \nu>,
$$

where $\nu$ is the outer unit normal vector on the lateral boundary of the tube $\Omega$.

Next step is to relate the perturbations of $\partial \Omega$ by means of $T_{\tau}(V)$ with perturbations of the curve $\gamma$ in the form $\gamma_{\varepsilon}$, where $\gamma_{\varepsilon}$ is a unit-speed parametrization of $\tilde{\gamma}_{\varepsilon}=\gamma+\varepsilon \beta$. We associate with $\Omega_{\varepsilon}=\Omega\left(\gamma_{\varepsilon}\right)$ the vector field $V(\beta)$ for $\varepsilon=0$. To this end, for a given parametrization $X_{\varepsilon}$ of $\partial \Omega_{\varepsilon}$ for $\varepsilon \geq 0$ we just have to evaluate

$$
V(\beta)=\lim _{\varepsilon \searrow 0} \frac{1}{\varepsilon}\left(X_{\varepsilon}-X\right) .
$$

The field is defined on $\partial \Omega$ and we have

$$
\lim _{\varepsilon \searrow 0} \frac{1}{\varepsilon}\left(J\left(\Omega_{\varepsilon}\right)-J(\Omega)\right)=d J(\Omega ; V(\beta))=<g_{\partial \Omega}, V(\beta) \cdot \nu>.
$$

Therefore, we can use the shape derivative $d J(\Omega ; V)$ in order to evaluate

$$
d I(\gamma ; \beta)=\lim _{\varepsilon \searrow 0} \frac{1}{\varepsilon}\left(I\left(\gamma_{\varepsilon}\right)-I(\gamma)\right)=<g_{\partial \Omega}, V(\beta) \cdot \nu>
$$

This means that knowing the form of the shape gradient $g_{\partial \Omega}$ for $J(\Omega)$ and of the speed vector field $V(\beta)$ on $\partial \Omega$ associated with the deformations $\Omega_{\varepsilon}=\Omega\left(\gamma_{\varepsilon}\right)$ of the tube $\Omega=\Omega(\gamma)$, we can evaluate the directional derivative $d I(\gamma ; \beta)$ and derive the optimality conditions. In the same way, we can obtain the second order derivative of $I(\gamma)$ (for example for Newton's method).

Our main result is

Theorem 5.1 Assume (H1)-(H6) then there exists an optimal curve $\gamma^{*}$ and an optimal domain $\Omega^{*}=$ $\Omega\left(\gamma^{*}\right)$, such that the following optimality system is satisfied:

(1) The state equations (2.10), (2.11), (2.16), (2.17) written with $\Omega^{*}=\Omega\left(\gamma^{*}\right)$.

(2) The adjoint state equations:

Find $(\eta, B, p, r)$ such that for all $\varphi \in H^{1}\left(\Omega^{*}\right), \psi \in \mathbf{X}$ and $\xi \in H^{1}(\Sigma)$,

$$
\begin{aligned}
& \int_{\Omega^{*}} \nabla \eta \cdot \nabla \varphi d x+\sigma_{0} \int_{\Omega^{*}} B \cdot \nabla \varphi d x=0, \quad \text { a.e. in }(0, T) \\
& B(T)=0, \quad \text { in } \Sigma \\
& -\sigma_{0} \int_{G^{*}} B_{t} \cdot \psi d x+\int_{D} \frac{1}{\mu} \operatorname{curl} B \cdot \operatorname{curl} \psi d x \\
& -2 \sigma_{0} \int_{\Sigma}\left(p A_{t}\right)_{t} \cdot \psi=0, \quad \text { a.e. in }(0, T)
\end{aligned}
$$




$$
\begin{aligned}
& p(T)=0, \quad \text { in } \Sigma \\
& -\rho c_{p} \int_{\Sigma} p_{t} \xi d x+k \int_{\Sigma} \nabla p \cdot \nabla \xi d x-\int_{\Sigma} \frac{\partial f}{\partial \theta} r \xi=0, \quad \text { a.e. in }(0, T) \\
& r(T, x)+2(z(T, x)-\bar{z}(x))=0, \quad \text { in } \Sigma \\
& -r_{t}-\rho L p_{t}-\frac{\partial f}{\partial z} r=0, \quad \text { in } \Sigma \times(0, T) .
\end{aligned}
$$

(3) The optimality condition

$$
\begin{aligned}
\int_{0}^{T} \int_{\partial \Omega^{*}} \nabla \varphi \cdot \nabla \eta V(h(\beta)) \cdot \nu d x d t & +\sigma_{0} \int_{0}^{T} \int_{\partial \Omega^{*}} A_{t} \cdot B V(h(\beta)) \cdot \nu d x d t+ \\
& +\int_{0}^{T} \int_{\partial \Omega^{*}} \nabla \varphi \cdot B V(h(\beta)) \cdot \nu d x d t+ \\
& +\int_{0}^{T} \int_{\partial \Omega^{*}}\left[\frac{1}{\mu} \operatorname{curl} A\right]_{\partial \Omega^{*}} \cdot \operatorname{curl} B V(h(\beta)) \cdot \nu d x d t \geq 0,
\end{aligned}
$$

for all $\beta \in T_{\mathcal{U}_{a d}}\left(\gamma^{*}\right)$, where $V(h)$ is the speed of $\partial \Omega_{\varepsilon}$ at $\varepsilon=0$ in the direction $h=h(\beta)$ given by

$$
h(\beta)(s)=\beta(s)-\left(\gamma^{*}\right)^{\prime}(s) \int_{0}^{s}\left(\gamma^{*}\right)^{\prime} \cdot \beta^{\prime} d \tilde{s} .
$$

$\beta$ is an admissible tangent direction, which defines the unit-speed perturbation $\gamma_{\varepsilon}$ of $\gamma^{*}$ by putting

$$
\tilde{\gamma}_{\epsilon}(s)=\gamma^{*}(s)+\varepsilon \beta(s), \quad 0 \leq s \leq l^{*}
$$

and

$$
\gamma_{\varepsilon}(s)=\tilde{\gamma}_{\varepsilon}\left(\tau_{\varepsilon}(s)\right)
$$

where the parameter transformation $\tau_{\varepsilon}$ is characterized by

$$
\left\{\begin{array}{l}
\left|\tau_{\varepsilon}^{\prime}(s)\right|=\left|\gamma^{\prime}\left(\tau_{\varepsilon}(s)\right)+\varepsilon \beta^{\prime}\left(\tau_{\varepsilon}(s)\right)\right|^{-1} \\
\tau_{\varepsilon}(0)=0
\end{array}\right.
$$

Admissible $\beta \in T_{\mathcal{U}_{a d}}\left(\gamma^{*}\right)$ satisfy the following conditions

(i) if $l^{*}=L_{1}$, then $\beta$ satisfies

$$
\left.\frac{\partial \tau_{\varepsilon}}{\partial \varepsilon}\right|_{\varepsilon=0}\left(L_{1}\right)=-\int_{0}^{L_{1}} \gamma^{\prime} \cdot \beta^{\prime} d s \geq 0
$$

if $l^{*}=L_{2}$, then $\beta$ satisfies

$$
\left.\frac{\partial \tau_{\varepsilon}}{\partial \varepsilon}\right|_{\varepsilon=0}\left(L_{2}\right)=-\int_{0}^{L_{2}} \gamma^{\prime} \cdot \beta^{\prime} d s \leq 0 ;
$$

(ii) if $\left\|\gamma^{*}\right\|_{H^{4}\left(0, l^{*}\right)}=M$, then $\beta$ satisfies

$$
(h(\beta), \gamma)_{H^{4}\left(0, l^{*}\right)} \leq 0 .
$$


Remark 5.1 Note that $\gamma_{\varepsilon}$ is admissible only, if reach $\left(\gamma_{\varepsilon}\right) \geq R+\delta$ for $\varepsilon>0$, small enough.

The proof of theorem 5.1 uses the following results on the shape differentiability of the shape functional $J(\Omega)$.

Theorem 5.2 Assume $(H 1)-H(6)$, then the shape fuctional $J(\Omega)$ is shape differentiable at any domain $\Omega \in \mathcal{U}_{a d}$

A direct consequence of Theorem 5.2 is that we can apply the structure theorem (cf. Theorem 2.27 in [17]) and obtain

Corollary 5.1 There exists a distribution $g_{\partial \Omega}$ supported on the lateral boundary $\Gamma_{2} \subset \partial \Omega$ such that

$$
d J(\Omega ; V)=<g_{\partial \Omega}, V \cdot \nu>
$$

\subsection{Proof of Theorem 5.2}

For a given admissible domain $\Omega \in \mathcal{U}_{a d}$ and a speed vector field

$$
V \in C^{1}\left(-\tau_{1}, \tau_{1} ; C^{2}\left(\mathbb{R}^{3} ; \mathbb{R}^{3}\right)\right),
$$

such that $\operatorname{supp} V \supset \bar{\Omega}$ and $\operatorname{supp} V \cap \Sigma=\emptyset$, we denote

$$
\Omega_{\tau}=T_{\tau}(V)(\Omega), \quad \tau \in\left[0, \tau_{1}\right]
$$

the family of domains associated with the flow of the field $V$, in particular $\Omega_{0}=\Omega$. All equations defined in $\Omega_{\tau}$ can be transported to the fixed domain $\Omega$ using the transformation $T_{\tau}^{-1}: \Omega_{\tau} \rightarrow \Omega$.

In the sequel we indicate functions on $\Omega_{\tau}$ with subscript $\tau$ and functions transported to the fixed domain $\Omega$ with superscript $\tau$, i.e. $f^{\tau}=f_{\tau} \circ T_{\tau}$.

The following lemma describes the transport of div and grad to the fixed domain. The proof can be found in [17], Sec. 2 .

Lemma 5.1 Let $B_{1}(\tau)^{-1}=\operatorname{det}\left(D T_{\tau}\right) D T_{\tau}^{-1}$, then we have

$$
(\operatorname{grad} \varphi) \circ T_{\tau}={ }^{*} D T_{\tau}^{-1} \cdot \operatorname{grad}\left(\varphi \circ T_{\tau}\right) \quad \text { for all } \varphi \in H^{1}\left(\mathbb{R}^{N}\right)
$$

$$
(\operatorname{div} \psi) \circ T_{\tau}=\frac{1}{\operatorname{det}\left(D T_{\tau}\right)} \operatorname{div}\left(B_{1}(\tau)^{-1}\left(\psi \circ T_{\tau}\right)\right), \quad \text { for all } \psi \in \mathbf{H}^{1}(\Omega) \text {. }
$$

Using Lemma 5.1, we obtain for (2.11) and $u \in H^{1}\left(\Omega_{\tau}\right)$

$$
\begin{aligned}
-\int_{\Gamma_{1}} j_{g} u d x & =\sigma_{0} \int_{\Omega_{\tau}} \nabla \phi_{\tau} \cdot \nabla u d x \\
& =\sigma_{0} \int_{\Omega} \operatorname{det}\left(D T_{\tau}\right)\left(\nabla \phi_{\tau} \cdot \nabla u\right) \circ T_{\tau} d x \\
& =\sigma_{0} \int_{\Omega} B_{2}(\tau) \nabla \phi^{\tau} \nabla\left(u \circ T_{\tau}\right) d x
\end{aligned}
$$

with

$$
B_{2}(\tau)=\beta(\tau) D T_{\tau}^{-1 *} D T_{\tau}^{-1} \quad \text { and } \quad \beta(\tau)=\operatorname{det}\left(D T_{\tau}\right)
$$

RR $\mathrm{n}^{\circ} 3782$ 
Hence, (2.11) is replaced with

$$
-\int_{\Gamma_{1}} j_{g} u d x=\alpha_{0}\left(\tau, \phi^{\tau}, u\right), \quad \text { for all } u \in H^{1}(\Omega),
$$

and

$$
\alpha_{0}\left(\tau, \phi^{\tau}, u\right)=\sigma_{0} \int_{\Omega} B_{2}(\tau) \nabla \phi^{\tau} \cdot \nabla u d x .
$$

For the Maxwell equation (2.10) the situation is more complicated, since functions $A_{\tau}$ that are divergence free on $\Omega_{\tau}$ generally loose this property when transported to the fixed domain. Therefore we introduce an auxiliary unknown function

$$
\eta^{\tau}=B_{1}(\tau)^{-1} A^{\tau}
$$

for which we have (cf. Lemma 5.1(2))

$$
\operatorname{div} A_{\tau}=0 \quad \text { in } \Omega_{\tau} \quad \Longleftrightarrow \quad \operatorname{div} \eta^{\tau}=0 \quad \text { in } \Omega .
$$

In the same manner we replace the test functions $v$ in $\Omega_{\tau}$ with

$$
w=B_{1}(\tau)^{-1} v \circ T_{\tau} \quad \text { in } \Omega .
$$

Next we transport the curl operater to the fixed domain. Let $e_{i}$ be the unit vector in $x_{i}$ direction, then we may write

$$
\operatorname{curl} A=\left(\operatorname{div}\left(A \times e_{1}\right), \operatorname{div}\left(A \times e_{2}\right), \operatorname{div}\left(A \times e_{3}\right)\right)^{T}
$$

and using Lemma 5.1, we obtain

$$
\begin{aligned}
\left(\operatorname{curl} A_{\tau}\right) \circ T_{\tau}= & \frac{1}{\operatorname{det}\left(D T_{\tau}\right)}\left(\operatorname{div}\left[B_{1}(\tau)^{-1}\left(A^{\tau} \times e_{1}\right)\right]\right. \\
& \left.\operatorname{div}\left[B_{1}(\tau)^{-1}\left(A^{\tau} \times e_{2}\right)\right], \operatorname{div}\left[B_{1}(\tau)^{-1}\left(A^{\tau} \times e_{3}\right)\right]\right)^{T} .
\end{aligned}
$$

Invoking (5.6) and (5.7) we obtain for (2.10):

$$
\begin{aligned}
\sigma_{0} \int_{G_{\tau}} \frac{\partial A_{\tau}}{\partial t} v d x & =\sigma_{0} \int_{G} \operatorname{det}\left(D T_{\tau}\right) A_{t}^{\tau} \cdot v \circ T_{\tau} d x \\
& =\sigma_{0} \int_{G} B_{3}(\tau) \eta_{t}^{\tau} \cdot w d x=\alpha_{1}\left(\tau, \eta_{t}^{\tau}, w\right),
\end{aligned}
$$

with

$$
B_{3}(\tau)=\operatorname{det}\left(D T_{\tau}\right){ }^{*} B_{1}(\tau) B_{1}(\tau)=\frac{1}{\operatorname{det}\left(D T_{\tau}\right)}{ }^{*} D T_{\tau} D T_{\tau}
$$

Using Einstein's summation convention, we get for the next term

$$
\begin{aligned}
\int_{D} \frac{1}{\mu} \operatorname{curl} A_{\tau} \cdot \operatorname{curl} v d x & \left.=\int_{D} \frac{\operatorname{det}\left(D T_{\tau}\right)}{\mu}\left(\operatorname{div}\left(A_{\tau} \times e_{i}\right) \operatorname{div} v \times e_{i}\right)\right) \circ T_{\tau} d x \\
& =\int_{D} \frac{1}{\beta(\tau) \mu} \operatorname{div}\left[B_{1}(\tau)^{-1}\left(A^{\tau} \times e_{i}\right)\right] \operatorname{div}\left[B_{1}(\tau)^{-1}\left(v \circ T_{\tau} \times e_{i}\right)\right] d x \\
& =\int_{D} \frac{1}{\beta(\tau) \mu} \operatorname{div}\left[B_{1}(\tau)^{-1}\left(B_{1}(\tau) \eta^{\tau} \times e_{i}\right)\right] \operatorname{div}\left[B_{1}(\tau)^{-1}\left(B_{1}(\tau) w \times e_{i}\right)\right] d x \\
& =\alpha_{2}\left(\tau, \eta^{\tau}, w\right) .
\end{aligned}
$$


For the last term in (2.10) we have

$$
\begin{aligned}
\int_{\Omega_{\tau}} \nabla \phi_{\tau} \cdot v d x & =\int_{\Omega} \operatorname{det}\left(D T_{\tau}\right)\left(\nabla \phi_{\tau} \cdot v\right) \circ T_{\tau} d x \\
& =\int_{\Omega} \operatorname{det}\left(D T_{\tau}\right)^{*} D T_{\tau}^{-1} \nabla \phi^{\tau} \cdot B_{1}(\tau) w d x \\
& =\int_{\Omega} \nabla \phi^{\tau} \cdot w d x=F\left(\nabla \phi^{\tau}, w\right) .
\end{aligned}
$$

Altogether, we have replaced (2.10) with

$$
\begin{gathered}
\alpha_{1}\left(\tau, \eta_{t}^{\tau}, w\right)+\alpha_{2}\left(\tau, \eta^{\tau}, w\right)+F\left(\phi^{\tau}, w\right)=0, \quad \text { for all } w \in X, \\
\eta_{0}^{\tau}=B_{1}(\tau)^{-1} A_{0} \circ T_{\tau} .
\end{gathered}
$$

With these preparations we can derive the material derivatives. We begin with

Lemma $5.2 B_{1}, B_{2}, B_{3}, \beta$ are differentiable. The derivatives at $\tau=0$ are given by

$$
\begin{aligned}
\beta^{\prime}(0) & =\operatorname{div} V(0), \\
B_{1}^{\prime}(0) & =-\operatorname{div} V(0) I+D V(0), \\
B_{2}^{\prime}(0) & =\operatorname{div} V(0) I-2 \varepsilon(V(0)), \\
B_{3}^{\prime}(0) & =-\operatorname{div} V(0) I+2 \varepsilon(V(0)) .
\end{aligned}
$$

Here, $\varepsilon(V(0))$ is the symmetrized part of $D V(0)$, i.e. $\varepsilon(V(0))=\frac{1}{2}\left(D V(0)+{ }^{*} D V(0)\right)$. For the proof we refer again to [17], Sec. 2.13.

Corollary 5.2 For $\tau>0$ small enough, we have

$$
\begin{aligned}
\beta(\tau) & =1+\tau \beta^{\prime}(0)+o(\tau) \\
B_{i}(\tau) & =I+\tau B_{i}^{\prime}(0)+o(\tau), \quad i=1,2,3 .
\end{aligned}
$$

A particular consequence of Corollary 5.1 is

Corollary 5.3 Let $\tau>0$ be small enough and $g_{i}$ be real-valued functions satisfying $g_{i}(\tau)=o(\tau), \quad i=$ $0,1,2$.

(1) For all $u_{1}, u_{2} \in H^{1}(\Omega)$ we have

$$
\begin{aligned}
\alpha_{0}\left(\tau, u_{1}, u_{2}\right) & =\alpha_{0}\left(0, u_{1}, u_{2}\right)+\tau \alpha_{0, \tau}\left(0, u_{1}, u_{2}\right)+\tilde{\alpha}_{0}\left(\tau, u_{1}, u_{2}\right), \\
\alpha_{0, \tau}\left(0, u_{1}, u_{2}\right) & =\int_{\Omega} B_{2}^{\prime}(0) \nabla u_{1} \nabla u_{2} d x \\
\left|\tilde{\alpha}_{0}\left(\tau, u_{1}, u_{2}\right)\right| & \leq g_{0}(\tau)\left\|\nabla u_{1}\right\|_{\mathbf{L}^{2}(\Omega)}\left\|\nabla u_{2}\right\|_{\mathbf{L}^{2}(\Omega)}
\end{aligned}
$$

(2) For all $w_{1}, w_{2} \in \mathbf{L}^{2}(D)$, we have

$$
\begin{aligned}
\alpha_{1}\left(\tau, w_{1}, w_{2}\right) & =\alpha_{1}\left(0, w_{1}, w_{2}\right)+\tau \alpha_{1, \tau}\left(0, w_{1}, w_{2}\right)+\tilde{\alpha}_{1}\left(\tau, w_{1}, w_{2}\right) \\
\alpha_{1, \tau}\left(0, w_{1}, w_{2}\right) & =\int_{G} B_{3}^{\prime}(0) w_{1} \cdot w_{2} d x \\
\left|\tilde{\alpha}_{1}\left(\tau, w_{1}, w_{2}\right)\right| & \leq g_{1}(\tau)\left\|w_{1}\right\|_{\mathbf{L}^{2}(\Omega)}\left\|w_{2}\right\|_{\mathbf{L}^{2}(\Omega)},
\end{aligned}
$$

$\mathrm{RR} \mathrm{n}^{\circ} 3782$ 
(3) For all $w_{1}, w_{2} \in \mathbf{X}$, we have

$$
\begin{aligned}
\alpha_{2}\left(\tau, w_{1}, w_{2}\right)= & \alpha_{2}\left(0, w_{1}, w_{2}\right)+\tau \alpha_{2, \tau}\left(0, w_{1}, w_{2}\right)+\tilde{\alpha}_{2}\left(\tau, w_{1}, w_{2}\right), \\
\alpha_{2, \tau}\left(0, w_{1}, w_{2}\right)= & -\int_{D} \frac{\beta^{\prime}(0)}{\mu} \operatorname{curl} w_{1} \cdot \operatorname{curl} w_{2} d x+\int_{D} \frac{1}{\mu} \operatorname{div}\left[\left(B_{1}^{-1}\right)^{\prime}(0)\left(w_{1} \times e_{i}\right)\right]\left(\operatorname{curl} w_{2}\right)_{i} d x \\
& +\int_{D} \frac{1}{\mu} \operatorname{div}\left[B_{1}^{\prime}(0) w_{1} \times e_{i}\right]\left(\operatorname{curl} w_{2}\right)_{i} d x \\
& +\int_{D} \frac{1}{\mu}\left(\operatorname{curl} w_{1}\right)_{i} \operatorname{div}\left[\left(B_{1}^{-1}\right)^{\prime}(0)\left(w_{2} \times e_{i}\right)\right] d x \\
& +\int_{D} \frac{1}{\mu}\left(\operatorname{curl} w_{1}\right)_{i} \operatorname{div}\left[B_{1}^{\prime}(0) w_{1} \times e_{i}\right] d x, \\
\left|\tilde{\alpha}_{2}\left(\tau, w_{1}, w_{2}\right)\right| \leq & g_{2}(\tau)\left\|w_{1}\right\|_{\mathbf{x}}\left\|w_{2}\right\|_{\mathbf{x}} \cdot
\end{aligned}
$$

Using Corollary 5.1, we can prove

\section{Lemma 5.3 (Stability)}

Assume (H1)-(H6), then there exists a constant $C>0$ such that

(1) $\left\|\nabla \phi^{\tau}-\phi\right\|_{H^{1}\left(0, T ; \mathbf{L}^{2}(\Omega)\right)} \leq C \cdot|\tau|$,

(2) $\left\|\eta^{\tau}-A\right\|_{L^{2}(0, T ; \mathbf{X})}+\left\|\eta_{t}^{\tau}-A_{t}\right\|_{L^{4}\left(0, T ; \mathbf{L}^{4}(G)\right)} \leq C \cdot|\tau|$,

(3) $\left\|z^{\tau}-z\right\|_{H^{1}\left(0, T ; L^{2}(\Sigma)\right) \cap L^{\infty}\left(0, T ; L^{4}(\Sigma)\right)} \leq C \cdot|\tau|$,

(4) $\left\|\theta^{\tau}-\theta\right\|_{H^{2,1}(Q)} \leq C \cdot|\tau|$.

Remark $5.2\left(z^{\tau}, \theta^{\tau}\right)$ is the solution to (2.16),(2.17) where $A_{t}$ in (2.17a) has been replaced with $\eta_{t}^{\tau}$. In view of (5.5) we have $\eta_{t}^{\tau}=A_{t}^{\tau}=A_{\tau, t}$ on $\Sigma$.

Proof: According to Lemma 5.1 we have $B_{2}(\tau)=I+\tau B_{2}^{\prime}(\xi)$ for $\tau$ small enough and $\xi \in(0, \tau)$. Using (H1) this gives immediately

$$
\left\|\nabla \phi^{\tau}\right\|_{H^{1}\left(0, T ; \mathbf{L}^{2}(\Omega)\right)} \leq c_{1}
$$

independent of $\tau$. Moreover, we have

$$
\begin{aligned}
0=\alpha_{0}\left(\tau, \phi^{\tau}, u\right) & -\alpha_{0}(0, \phi, u) \\
& =\alpha_{0}\left(0, \phi^{\tau}-\phi, u\right)+\tau \int_{\Omega} B_{2}^{\prime}(\xi) \phi^{\tau} \nabla \phi^{\tau} \cdot \nabla u d x .
\end{aligned}
$$

Inserting $u=\phi^{\tau}-\phi$ and using Young's inequality we obtain

$$
\left\|\nabla \phi^{\tau}-\nabla \phi\right\|_{L^{2}\left(0, T ; \mathbf{L}^{2}(\Omega)\right)} \leq c_{2}|\tau|
$$

Since the same estimate holds true for $\phi_{t}^{\tau}-\phi_{t}$, assertion (1) is proved. In the same way, writing $B_{1}(\tau)=I+\tau B_{1}^{\prime}(\xi), \quad B_{3}(\tau)=I+\tau B_{3}^{\prime}(\xi)$, (note that $\left(B_{1}^{-1}\right)^{\prime}=-B_{1}^{\prime}$ ), we obtain

$$
\left\|\eta^{\tau}\right\|_{L^{2}(0, T ; \mathbf{X})} \leq c_{3},
$$

independent of $\tau$, and, defining

$$
\eta_{0, t}^{\tau}=B_{1}(\tau)^{-1} y \circ T_{\tau}
$$

(cf. (H3) and (5.8b)), we get

$$
\left\|\eta_{t}^{\tau}\right\|_{L^{4}\left(0, T ; \mathbf{L}^{4}(G)\right)} \leq c_{4} .
$$


Now we take the difference of (5.8) for $\eta^{\tau}$ and $A$ and obtain

$$
\begin{aligned}
0 & =\alpha_{1}\left(\tau, \eta_{t}^{\tau}, w\right)+\alpha_{2}\left(\tau, \eta^{\tau}, w\right)+F\left(\phi^{\tau}, w\right)-\alpha_{1}\left(0, A_{t}, w\right)-\alpha_{2}(0, A, w)-F(\phi, w) \\
& =\alpha_{1}\left(0, \eta_{t}^{\tau}-A_{t}, w\right)+\alpha_{2}\left(0, \eta^{\tau}-A, w\right)+F\left(\phi^{\tau}-\phi, w\right)+\tau \int_{G} B_{3}^{\prime}(\xi) \eta_{t}^{\tau} w d x+\tau G\left(\eta^{\tau}, w\right)
\end{aligned}
$$

with a function $G$ that satisfies

$$
\left|G\left(w_{1}, w_{2}\right)\right| \leq c_{5}\left\|w_{1}\right\|_{\mathbf{x}} \cdot\left\|w_{2}\right\|_{\mathbf{x}}
$$

Putting $w=\eta^{\tau}-A$ and integrating in time leads to

$$
\begin{aligned}
& \frac{\sigma_{0}}{2} \int_{G}\left|\eta^{\tau}(t)-A(t)\right|^{2} d x+\int_{0}^{t} \int_{D} \frac{1}{\mu}\left|\operatorname{curl}\left(\eta^{\tau}-A\right)\right|^{2} d x d t \\
& \quad \leq \int_{0}^{t} \int_{D}\left|\nabla\left(\phi^{\tau}-\phi\right) \cdot\left(\eta^{\tau}-A\right)\right| d x d t+\frac{\sigma_{0}}{2} \int_{G}\left|\eta_{0}^{\tau}-A_{0}\right|^{2} d x \\
& \quad+|\tau| c_{5} \int_{0}^{t}\left\|\eta^{\tau}\right\|_{\mathbf{X}} \cdot\left\|\eta^{\tau}-A\right\|_{\mathbf{X}}+|\tau| c_{6} \int_{0}^{t}\left\|\eta_{t}^{\tau}\right\|_{\mathbf{L}^{2}(G)} \cdot\left\|\eta^{\tau}-A\right\|_{\mathbf{L}^{2}(G)}
\end{aligned}
$$

Applying the inequalities of Young and Gronwall and using (5.8b) the first part of assertion (2) is proved. Differentiating (5.8a) formally with respect to time, making the same computations as before but using (5.9) finishes the proof of assertion (2).

Now, we test the difference of (2.16) for $z^{\tau}$ and $z$ with $\bar{z}_{t}=z_{t}^{\tau}-z_{t}$. Using (H4)-(H6) and Young's inequality, defining $\bar{\theta}=\theta^{\tau}-\theta$, we obtain

$$
\begin{aligned}
\frac{1}{2} \int_{0}^{t} \int_{\Sigma} \bar{z}_{s}^{2} d x & \leq \frac{1}{2} \int_{0}^{t} \int_{\Sigma}\left(\frac{\left[b\left(\theta^{\tau}\right)-z^{\tau}\right]^{+}}{a\left(\theta^{\tau}\right)}-\frac{[b(\theta)-z]^{+}}{a(\theta)}\right)^{2} d x d s \\
& \leq c_{7} \int_{0}^{t} \int_{\Sigma} \bar{\theta}^{2} d x d s+c_{8} \int_{0}^{t} \int_{\Sigma} \bar{z}^{2} d x d s
\end{aligned}
$$

Invoking Gronwall's inequality leads to

$$
\|\bar{z}\|_{H^{1}\left(0, T ; L^{2}(\Sigma)\right)} \leq c_{9}\|\bar{\theta}\|_{L^{2}(Q)} .
$$

Next, we test the difference of (2.17) for $\theta^{\tau}$ and $\theta$ with $\bar{\theta}_{t}$ and apply the inequalities of Hölder and Young to get:

$$
\begin{aligned}
& \rho c_{p} \int_{0}^{t} \int_{\Sigma} \bar{\theta}_{s}^{2} d x d s+\frac{k}{2} \int_{\Sigma}|\nabla \bar{\theta}(t)|^{2} d x d s \\
& \quad \leq \rho L \int_{0}^{t} \int_{\Sigma} \bar{z}_{s} \bar{\theta}_{s} d x d s+\sigma_{0} \int_{0}^{t} \int_{\Sigma}\left|\left(\eta_{s}^{\tau}-A_{s}\right) \cdot\left(\eta_{s}^{\tau}+A_{s}\right)\right|^{2} d x d s \\
& \leq \frac{\rho c_{p}}{2} \int_{0}^{t} \int_{\Sigma} \bar{\theta}_{s}^{2} d x d s+c_{10} \int_{0}^{t}\|\bar{\theta}\|_{L^{2}(\Sigma)}^{2}+c_{11} \int_{0}^{t}\left\|\eta_{s}^{\tau}-A_{s}\right\|_{L^{4}(\Sigma)}^{2}\left\|\eta_{s}^{\tau}+A_{s}\right\|_{L^{4}(\Sigma)}^{2} .
\end{aligned}
$$

Using (5.11), assertion (2) and Gronwall's inequality, we get

$\mathrm{RR} \mathrm{n}^{\circ} 3782$

$$
\|\bar{\theta}\|_{L^{\infty}\left(0, T ; H^{1}(\Sigma)\right) \cap H^{1}\left(0, T ; L^{2}(\Sigma)\right)} \leq c_{12}|\tau| .
$$


In view of the last inequality we can test the difference of (2.16) for $z^{\tau}$ and $z$ with $\bar{z}^{3}$, apply the inequalities of Young and Gronwall and obtain

$$
\|\bar{z}\|_{L^{\infty}\left(0, T ; L^{4}(\Sigma)\right)} \leq c_{13}|\tau| .
$$

Testing (2.17) with $-\Delta \bar{\theta}$ making the same computations as above finishes the proof.

Remark 5.3 All the unknowns depend on the shape of $\Omega_{\tau}$, either explicitly as $A^{\tau}$ and $\phi^{\tau}$ or implicitly as $\theta^{\tau}$ and $z^{\tau}$. For all these quantities, we call

$$
\dot{f}=\lim _{\tau \rightarrow 0} \frac{f^{\tau}-f}{\tau}
$$

the strong material derivative of $f$, whenever the limit exists in the strong sense.

Our main result in this subsection is

Lemma 5.4 (Strong material derivatives)

Assume (H1)-(H6), then the following are valid:

(1) The strong material derivative

$\nabla \dot{\phi}$ exists in $H^{1}\left(0, T ; \mathbf{L}^{2}(\Omega)\right)$,

$\dot{A}$ exists in $L^{\infty}(0, T ; X)$ and $W^{1,4}\left(0, T ; L^{4}(G)\right)$,

$\dot{z}$ exists in $H^{1}\left(0, T ; L^{2}(\Sigma)\right)$,

$\dot{\theta}$ exists in $H^{2,1}(Q)$.

(2) Moreover, $(\dot{\phi}, \dot{A}, \dot{z}, \dot{\theta})$ satisfy the linearized state equations

$$
\begin{aligned}
\alpha_{0}(0, \dot{\phi}, u)+\alpha_{0, \tau}(0, \phi, u) & =0, \quad \text { for all } u \in H^{1}(\Omega), \\
\alpha_{1}\left(0, \dot{A}_{t}, w\right)+\alpha_{2}(0, \dot{A}, w)+F(\dot{\phi}, w) & \\
+\alpha_{1, \tau}\left(0, A_{t}, w\right)+\alpha_{2, \tau}(0, A, w) & =0, \quad \text { for all } w \in \mathbf{X}, \\
\dot{A}(0)-D A_{0} V(0)+B_{1}^{\prime}(0) A_{0} & =0, \quad \text { in } D, \\
\dot{z}_{t}-\frac{\partial f}{\partial \theta} \dot{\theta}-\frac{\partial f}{\partial z} \dot{z} & =0, \quad \text { in } Q, \\
\dot{z}(0) & =0, \quad \text { in } \Sigma, \\
\rho c_{p} \dot{\theta}_{t}-k \Delta \dot{\theta}+\rho L \dot{z}_{t}-2 \sigma_{0} A_{t} \cdot \dot{A}_{t} & =0, \quad \text { in } Q, \\
\frac{\partial \dot{\theta}}{\partial \nu} & =0, \quad \text { in } \partial \Sigma \times(0, T), \\
\dot{\theta}(0) & =0, \quad \text { in } \Sigma,
\end{aligned}
$$

where $f$ is the right-hand side of (2.16b).

Proof:

Similar to the proofs of Lemma 2.1-2.3 one can show that (5.11a-i) has a solution and that $(\nabla \dot{\phi}, \dot{A}, \dot{z}, \dot{\theta})$ are uniquely defined. It remains to show that these solutions are the strong material derivatives. To this end let

$$
\psi^{\tau}=\frac{1}{\tau}\left(\phi^{\tau}-\phi\right)-\dot{\phi}
$$

then according to Corollary 5.3(1), (5.4) and (5.11a), $\psi^{\tau}$ satisfies

$$
\begin{aligned}
\alpha_{0}\left(0, \psi^{\tau}, u\right) & =-\frac{1}{\tau}\left(\alpha_{0}\left(\tau, \phi^{\tau}, u\right)-\alpha_{0}\left(0, \phi^{\tau}, u\right)\right)-\alpha_{0}(0, \dot{\phi}, u) \\
& =\alpha_{0, \tau}\left(0, \phi-\phi^{\tau}, u\right)-\frac{1}{\tau} \tilde{\alpha}_{0}\left(0, \phi^{\tau}, u\right)
\end{aligned}
$$


Integrating in time, inserting $u=\psi^{\tau}$ and using Corollary 5.3(1) once again we obtain

$$
\left\|\nabla \psi^{\tau}\right\|_{L^{2}\left(0, T ; \mathbf{L}^{2}(\Omega)\right)}=O(\tau) .
$$

Since the same computations hold for $\nabla \phi_{t}$, the first part of assertions (1) and (2) is proved.

Next, defining

$$
p^{\tau}=\frac{1}{\tau}\left(\eta^{\tau}-A\right)-\dot{A},
$$

and using (5.12) and Corollary 5.3, we see that $p^{\tau}$ satisfies

$$
\begin{aligned}
& \alpha_{1}\left(0, q_{t}^{\tau}, w\right)+\alpha_{2}\left(0, p^{\tau}, w\right)=-\frac{1}{\tau}\left(F\left(\phi^{\tau}, w\right)-F(\phi, w)\right) \\
&-\frac{1}{\tau}\left(\alpha_{1}\left(\tau, \eta_{t}^{\tau}, w\right)-\alpha_{1}\left(0, \eta_{t}^{\tau}, w\right)\right)-\frac{1}{\tau}\left(\alpha_{2}\left(\tau, \eta^{\tau}, w\right)-\alpha_{2}\left(0, \eta^{\tau}, w\right)\right) \\
&+F(\dot{\phi}, w)+\alpha_{1, \tau}\left(0, A_{t}, w\right)+\alpha_{2, \tau}(0, A, w) \\
&\left.=-F\left(\psi^{\tau}, w\right)-\alpha_{1, \tau}\left(0, \eta_{t}^{\tau}-A_{t}, w\right)-\alpha_{2, \tau}\left(0, \eta^{\tau}-A\right), w\right) \\
&-\frac{1}{\tau} \tilde{\alpha}_{1}\left(\tau, \eta_{t}^{\tau}, w\right)-\frac{1}{\tau} \tilde{\alpha}_{2}\left(\tau, \eta^{\tau}, w\right) .
\end{aligned}
$$

We take $w=p^{\tau}$ and integrate in time to obtain

$$
\begin{aligned}
& \frac{\sigma_{0}}{2} \int_{G}\left|p^{\tau}\right|^{2} d x-\frac{\sigma_{0}}{2} \int_{G}\left|p_{0}^{\tau}\right|^{2} d x+\int_{0}^{t} \int_{D} \frac{1}{\mu}\left|\operatorname{curl} p^{\tau}\right|^{2} d x d s \\
& \leq c_{1} \int_{0}^{t} \int_{G}\left|p^{\tau}\right|^{2} d x+\delta \int_{0}^{t} \int_{D} \frac{1}{\mu}\left|\operatorname{curl} p^{\tau}\right|^{2} d x d s+c_{2} \int_{0}^{t} \int_{G}\left|\eta_{t}^{\tau}-A_{t}\right|^{2} d x d s \\
& \quad+\frac{1}{4 \delta} \int_{0}^{t} \int_{D} \frac{1}{\mu}\left|\operatorname{curl} \eta^{\tau}-\operatorname{curl} A\right|^{2} d x d s+c_{3} \int_{0}^{t}\left|\nabla \psi_{G}^{\tau}\right|^{2} d x d s+O\left(\tau^{2}\right) .
\end{aligned}
$$

Using (5.8b), the second term in (5.13) gives

$$
\int_{G}\left|p_{0}^{\tau}\right|^{2} d x=\int_{G}\left|\frac{1}{\tau}\left(B_{1}(\tau)^{-1} A_{0} \circ T_{\tau}-A_{0}\right)-\dot{A}_{0}\right|^{2} d x .
$$

According to [17], Sec. 2.14, $\tau \mapsto A_{0} \circ T_{\tau}$ is differentiable with

$$
\left.\frac{d}{d \tau}\left(A_{0} \circ T_{\tau}\right)\right|_{\tau=0}=D A_{0} V(0)
$$

hence

$$
A_{0} \circ T_{\tau}=A_{0}+\tau D A_{0} V(0)+o(\tau) .
$$

Moreover, Corollary 5.2 implies

$$
B_{1}(\tau)^{-1}=I-\tau B_{1}^{\prime}(0)+O\left(\tau^{2}\right)
$$

Altogether, we obtain

$$
\int_{G}\left|p_{0}^{\tau}\right|^{2} d x=O\left(\tau^{2}\right)
$$

Taking $\delta$ small enough and invoking Gronwall's Lemma, we obtain from (5.13)

$\mathrm{RR} \mathrm{n}^{\circ} 3782$

$$
\left\|p^{\tau}\right\|_{L^{\infty}\left(0, T ; \mathbf{L}^{2}(G)\right)}^{2}+\int_{0}^{T}\left\|p^{\tau}\right\|_{\mathbf{X}}^{2}=O\left(\tau^{2}\right) .
$$


Now, we differentiate (5.8) formally with respect to time, using (5.9) as initial condition and make the same estimates as above. Reasoning similar to the end of the proof of Lemma 2.2, we obtain

$$
\left\|p_{t}^{\tau}\right\|_{L^{4}\left(0, T ; \mathbf{L}^{4}(G)\right)}=O(\tau) .
$$

To prove the differentiability of $\theta^{\tau}$ and $z^{\tau}$, we first remark that there exists a constant $C>0$, such that

$$
\|\dot{\theta}\|_{H^{2,1}(Q)}+\|\dot{z}\|_{H^{1}\left(0, T ; L^{2}(\Sigma)\right) \cap L^{\infty}\left(0, T ; L^{4}(\Sigma)\right)} \leq C .
$$

We define

$$
\begin{aligned}
q^{\tau} & =\frac{1}{\tau}\left(\theta^{\tau}-\theta\right)-\dot{\theta} \\
r^{\tau} & =\frac{1}{\tau}\left(z^{\tau}-z\right)-\dot{z}
\end{aligned}
$$

then, $\left(q^{\tau}, r^{\tau}\right)$ solve

$$
\begin{aligned}
\rho c_{p} q^{\tau}-k \Delta q^{\tau} & =-\rho L r^{\tau}+\sigma_{0} \tau\left|\dot{A}_{t}\right|^{2}+\sigma_{0} p_{t}^{\tau} \cdot\left(2 A_{t}+2 \tau \dot{a}_{t}+\tau p_{t}^{\tau}\right) \\
r^{\tau}= & \frac{1}{\tau}\left(f\left(\theta^{\tau}, z^{\tau}\right)+f(\theta, z)\right)-\frac{\partial f}{\partial \theta}(\theta, z) \dot{\theta}-\frac{\partial f}{\partial z}(\theta, z) \dot{z} \\
=: & G(\tau) \\
\frac{\partial q^{\tau}}{\partial \nu}=0, & q^{\tau}=0, \quad r^{\tau}(0)=0 .
\end{aligned}
$$

Owing to (H4)-(H6), we can apply Taylor's formula to develop $G(\tau)$ and obtain (with a constant $\xi \in[0,1])$

$$
\begin{aligned}
|G(\tau)|= & \left|\frac{1}{\tau}\left(f\left(\theta+\tau\left(q^{\tau}+\dot{\theta}\right), z+\tau\left(r^{\tau}+\dot{z}\right)\right)-f(\theta, z)\right)-\frac{\partial f}{\partial \theta}(\theta, z) \dot{\theta}-\frac{\partial f}{\partial z}(\theta, z) \dot{z}\right| \\
= & \mid\left(q^{\tau}+\dot{\theta}\right) \frac{\partial f}{\partial \theta}\left(\theta+\xi \tau\left(q^{\tau}+\dot{\theta}\right), z+\xi \tau\left(r^{\tau}+\dot{z}\right)\right) \\
& \quad+\left(r^{\tau}+\dot{z}\right) \frac{\partial f}{\partial z}\left(\theta+\xi \tau\left(q^{\tau}+\dot{\theta}\right), z+\xi \tau\left(r^{\tau}+\dot{z}\right)\right)-\frac{\partial f}{\partial \theta}(\theta, z) \dot{\theta}-\frac{\partial f}{\partial z}(\theta, z) \dot{z} \mid \\
\leq & c_{4}\left|q^{\tau}\right|+c_{5}\left|r^{\tau}\right|+|\dot{\theta}|\left|\frac{\partial f}{\partial \theta}\left(\theta+\xi \tau\left(q^{\tau}+\dot{\theta}\right), z+\xi \tau\left(r^{\tau}+\dot{z}\right)\right)-\frac{\partial f}{\partial \theta}(\theta, z)\right| \\
& \quad+|\dot{z}|\left|\frac{\partial f}{\partial z}\left(\theta+\xi \tau\left(q^{\tau}+\dot{\theta}\right), z+\xi \tau\left(r^{\tau}+\dot{z}\right)\right) \frac{\partial f}{\partial z}(\theta, z)\right| \\
\leq & \quad\left(c_{4}+c_{6}|\tau||\dot{\theta}|+c_{8}|\tau||\dot{z}|\right)\left|q^{\tau}\right|+|\tau|\left(c_{6} \dot{\theta}^{2}+c_{8}|\dot{\theta}||\dot{z}|\right)+\left(c_{5}+c_{7}|\tau||\dot{\theta}|\right)\left|r^{\tau}\right|+c_{9}|\dot{z}|\left|z^{\tau}-z\right| .
\end{aligned}
$$

Invoking Hölder's inequality, Sobolev's embedding theorem and Lemma 5.3(3), we can conclude

$$
\begin{aligned}
\int_{0}^{t}\|G(\tau)\|_{L^{2}(\Sigma)}^{2} d s \leq & O\left(\tau^{2}\right)+\int_{0}^{t}\left\|\left(c_{4}+c_{6}|\tau||\dot{\theta}|+c_{8}|\tau \| \dot{z}|\right)\right\|_{L^{4}(\Sigma)}^{2}\left\|q^{\tau}\right\|_{H^{1}(\Sigma)}^{2} d s \\
& +\int_{0}^{t}\left(c_{5}+c_{7}|\tau|\|\dot{\theta}\|_{H^{2}(\Sigma)}\right)\left\|r^{\tau}\right\|_{L^{2}(\Sigma)}^{2} d s .
\end{aligned}
$$

Now testing (5.15b) with $r_{t}^{\tau}$ and applying Gronwall's and Young's inequality leads to

$$
\frac{1}{2} \int_{0}^{t} \int_{\Sigma} r_{s}^{2} d x d s \leq O\left(\tau^{2}\right)+c_{1)} \int_{0}^{t}\left\|\left(c_{4}+c_{6}|\tau \| \dot{\theta}|+c_{8}|\tau||\dot{z}|\right)\right\|_{L^{4}(\Sigma)}^{2}\left\|q^{\tau}\right\|_{H^{1}(\Sigma)}^{2} d s .
$$


The proof is concluded by testing (5.15a) successively with $q_{t}^{\tau}$ and $-\Delta q^{\tau}$, taking into account the previous estimate as well as (5.14) and Gronwall's inequality.

Using Lemma 5.4, we see that the Eulerian derivative (5.1) exists and that it is linear and continuous with respect to $V$. This concludes the proof of Theorem 5.2.

\subsection{Proof of Theorem 5.1}

Let $\gamma$ be an optimal solution and $\gamma_{\varepsilon}=\gamma+\varepsilon h+o(\varepsilon)$ in $C^{3}$ be an admissible perturbation of the curve $\gamma$ (cf. Appendix). Then,

$$
I\left(\gamma_{\varepsilon}\right) \geq I(\gamma),
$$

where $I$ is defined in (3.4). In view of Theorem 5.1, we have

$$
J\left(\Omega\left(\gamma_{\varepsilon}\right)\right)=J(\Omega(\gamma))+\varepsilon d J(\Omega(\gamma) ; V(h))+o(\varepsilon) .
$$

Hence, from (5.16) it follows that

$$
d J(\Omega(\gamma) ; V(h)) \geq 0
$$

for all admissible directions $h \in C^{3}$; and in particular

$$
d I(\gamma ; h)=\lim _{\varepsilon \searrow 0} \frac{1}{\varepsilon}\left[I\left(\gamma_{\varepsilon}\right)-I(\gamma)\right]=d J(\Omega(\gamma) ; V(h))
$$

Applying the structure theorem (cf. Corollary 5.1) we obtain

$$
d I(\gamma ; h)=<g_{\partial \Omega} ; V(h) \cdot \nu>.
$$

In general, $g_{\partial \Omega}$ is a distribution. Assuming that the density $g_{\partial \Omega}$ is a function, it can be identified in the following way. Utilizing a general strategy to derive the first order optimality system described eg. by Cea in [4], we introduce the Lagrangean

$$
\begin{aligned}
& \mathcal{L}_{\varepsilon}(\phi, \eta ; A, B ; \theta, p, z, r)=J\left(\Omega_{\varepsilon}\right) \\
& \quad+\int_{0}^{T} \int_{\Omega_{\varepsilon}} \nabla \phi \cdot \nabla \eta d x d t-\int_{0}^{T} \int_{\Gamma_{1}} j \eta d x d t \\
& \quad+\int_{0}^{T} \int_{D}\left[\sigma_{0}^{\varepsilon} A_{t} \cdot B+\frac{1}{\mu_{\varepsilon}} \operatorname{curl} A \cdot \operatorname{curl} B\right] d x d t+\sigma_{0} \int_{0}^{T} \int_{\Omega_{\varepsilon}} \nabla \phi \cdot B d x d t \\
& \quad+\int_{0}^{T} \int_{\Sigma}\left[\rho c_{p} \theta_{t} p+k \nabla \theta \cdot \nabla p+\rho L z_{t} p-\sigma_{0}\left|A_{t}\right|^{2} p\right] d x d t \\
& \quad+\int_{0}^{T} \int_{\Sigma}\left[z_{t}-f(\theta, z)\right] r d x d t .
\end{aligned}
$$

Here, $\Omega_{\varepsilon}=\Omega\left(\gamma_{\varepsilon}\right)$ and $\sigma_{0}^{\varepsilon}=\sigma_{0}$ in $\Omega_{\varepsilon}$ and 0 otherwise.

The adjoint state equations are obtained for $\varepsilon=0$ by differentiation of the Langrangean with respect to the state variables, i.e. $\left\langle\frac{\partial \mathcal{L}_{0}}{\partial \phi} ; \varphi>=0\right.$ gives $(5.2 \mathrm{a}),<\frac{\partial \mathcal{L}_{0}}{\partial A} ; \psi>=0$ gives $(5.2 \mathrm{~b}, \mathrm{c}),<\frac{\partial \mathcal{L}_{0}}{\partial \theta} ; \xi>=0$ gives $(5.2 \mathrm{~d}, \mathrm{e}),<\frac{\partial \mathcal{L}_{0}}{\partial z} ; w>=0$ gives $(5.2 \mathrm{f}, \mathrm{g})$.

It is easy to see that the linear system of adjoint equations admits a unique solution. First of all, one proves that $(5.2 \mathrm{~d}-\mathrm{g})$ admits a unique strong solution $(p, r)$ by a contraction mapping argument in

$\mathrm{RR} \mathrm{n}^{\circ} 3782$ 
$H^{1}\left(0, T ; L^{2}(\Sigma)\right)$. Then, $(5.2 \mathrm{~b}, \mathrm{c})$ is solved using Lemma 2.2 and finally standard elliptic theory shows the solvability of $(5.2 \mathrm{a})$.

The gradient of the cost functional is given by the derivative

$$
d I(\gamma ; h)=d J(\Omega(\gamma) ; V(h))=\left.\frac{\partial \mathcal{L}_{\varepsilon}}{\partial \varepsilon}\right|_{\varepsilon=0},
$$

assuming that we have enough regularity for the solution to the state and the adjoint equations. To derive the gradient, we make use of the following

Lemma 5.5 (cf. Proposition 2.46 in [17])

Let $f \in W^{1,1}\left(\mathbb{R}^{3}\right)$ and $\Omega \subset \mathbb{R}^{3}$ with boundary of class $C^{k}, k \geq 1$, then

$$
\left.\left(\frac{\partial}{\partial \varepsilon} \int_{\Omega_{\varepsilon}} f d x\right)\right|_{\varepsilon=0}=\int_{\partial \Omega} f V \cdot \nu d x,
$$

where $\nu$ is the outer unit normal on $\partial \Omega$.

Using this lemma and taking into account the different values for permeability in the tube and in the air, we directly obtain (5.3), which concludes the proof of Theorem 5.1.

\section{Acknowledgement}

The authors gratefully acknowledge financial support by $D A A D$ (Germany) and by the Ministry of Foreign Affairs (France) within the scope of the French-German project PROCOPE No. 98158.

\section{Références}

[1] Arnăutu, V., Hömberg, D., Sokołowski, J, Convergence results for a nonlinear parabolic control problem, WIAS Preprint 490 (1999).

[2] Bossavit, A., Rodrigues, J.-F., On the electromagnetic "Induction Heating" Problem in bounded domains, Adv. Math. Sc. Appl., 4, (1994), 79-92.

[3] Bodart, O., Touzani, R., Optimal control of electromagnetic induction heating 1. a two-control parameter model, Preprint Laboratoire de Mathématiques Appliquées, Université Blaise Pascal (Clermont-Ferrand 2).

[4] Cea, J., Conception optimale ou identification de formes, calcul rapide de la dèrivèe directionelle de la fonction coût, Math. Mod. \& Num. Anal. 20 (1986), 371-402.

[5] Chenais, D. Sur une famille de variétés a bord Lipschitziennes, application à un problème d'identification de domaines, Ann. Inst. Fourier, Vol. 27, No. 4, (1977), 201-231.

[6] Clain, S. et al., Numerical modelling of induction heating for two-dimensional geometries, Math. Models Methods Appl. Sci., Vol. 3, No. 6 (1993), 271-281.

[7] Dautray, R., Lions, J.-L., Mathematical analysis and numerical methods for science and technology, Vol. 3, Springer-Verlag, Berlin 1990.

[8] Egan, L.R., Furlani, E. P., A computer simulation of an induction heating system, IEEE Trans. Mag. 27, No.5 (1991), 4343-4354.

[9] Federer, H., Curvature measures, Trans.-Amer.-Math.-Soc., 93 (1959), 418-491.

[10] Fuhrmann, J., Hömberg, D., Numerical simulation of surface heat treatments, Num. Meth. Heat $\&$ Fluid Flow.

[11] Gray, A., Modern differential geometry of curves and surfaces, CRC Press, Boca Raton, 1993.

[12] Hömberg, D., A mathematical model for the phase transitions in eutectoid carbon steel, IMA J. Appl. Math., 54 (1995), 31-57. 
[13] Hömberg, D., Sokołowski, J, Optimal control of laser heat treatments. Advances in Math. Sc. Appl. 8 (1998), 911-928.

[14] Hoppe, R.H.W., Kornhuber, R., Multi-grid solution of two coupled Stefan equations arising in induction heating of large steel slabs, Internat. J. Numer. Methods Engrg., 30, (1990), 779-801.

[15] Leblond, J.-B., Devaux, J.,A new kinetic model for anisothermal metallurgical transformations in steels including effect of austenite grain size, Acta Met. 32, (1984), 137-146.

[16] Kačur, J., Method of Rothe in Evolution Equations, Teubner-Texte zur Mathematik, Vol. 80, Leipzig 1985.

[17] Sokołowski, J., Zolesio, J.-P., Introduction to shape optimization, Springer series in computational mathematics, Vol. 16, Berlin 1992.

[18] Visintin, A., Mathematical models of solid-solid phase transitions in steel, IMA J. Appl. Math., 39 (1987), 143-157.

\section{A Calculation of the speed vector field}

In the appendix we explain, how the speed vector field $V(h(\beta))$ of $\partial \Omega\left(\gamma_{\varepsilon}\right)$ at $\varepsilon=0$ can be computed from a perturbation of the curve $\gamma$. To this end let $\tilde{\gamma}_{\varepsilon}=\gamma+\varepsilon \beta$, then $\gamma_{\varepsilon}$ is the unit-speed curve

$$
\gamma_{\varepsilon}(s)=\gamma\left(\tau_{\varepsilon}(s)\right)+\varepsilon \beta\left(\tau_{\varepsilon}(s)\right), \quad 0 \leq s \leq l_{\varepsilon}, \varepsilon \geq 0,
$$

where $\tau_{\varepsilon}:\left[0, l_{\varepsilon}\right] \rightarrow[0, l]$ is a reparametrization satisfying $\tau_{\varepsilon}(0)=0, \tau_{\varepsilon}\left(l_{\varepsilon}\right)=l$ and $\tau_{\varepsilon}^{\prime}(s)>0$ for all $s \in\left[0, l_{\varepsilon}\right]$.

We recall that the lateral tube boundary $\Gamma_{2}$ is parametrized by

$$
\Gamma_{2}=\left\{x \in \mathbb{R}^{3} \mid X(u, v)=\gamma(u)+R \cos v N(u)+R \sin v B(u), 0 \leq u \leq l, 0 \leq v<2 \pi\right\} .
$$

Using (A.1) and the unit-speed property of $\gamma_{\varepsilon}$, we have

$$
\gamma_{\varepsilon}=\gamma+\varepsilon h(\beta)+o(\varepsilon) \quad \text { in } C^{3}\left[0, l_{\varepsilon}\right]
$$

where

$$
h(\beta)(s)=\beta(s)-\gamma^{\prime}(s) \int_{0}^{s} \gamma^{\prime}(\xi) \cdot \beta^{\prime}(\xi) d \xi .
$$

If $l_{\varepsilon}>l$, we of course have to extend $\gamma$ in the same way than in the proof of Theorem 4.1.

We assume that the normal and binormal vector fields $N_{\varepsilon}(u), B_{\varepsilon}(u)$ take the form

$$
\begin{array}{ll}
N_{\varepsilon}=\mathbf{N}+\varepsilon N_{1}(h)+o(\varepsilon), & \text { in } C^{1}\left[0, l_{\varepsilon}\right], \\
B_{\varepsilon}=\mathbf{B}+\varepsilon B_{1}(h)+o(\varepsilon), & \text { in } C^{1}\left[0, l_{\varepsilon}\right] .
\end{array}
$$

Remark A.1 If $\left|\gamma^{\prime \prime}(u)\right|>0$ for all $u \in[0, l]$, then we have the Frenet Formulas

$$
\begin{aligned}
T^{\prime} & =\kappa N, \\
N^{\prime} & =-\kappa T+\tau B, \\
B^{\prime} & =-\tau N,
\end{aligned}
$$

where $\tau$ is the torsion, and the curve $\gamma_{\varepsilon}$ defines in a unique way the fields $N_{\varepsilon}(u), B_{\varepsilon}(u)$. 
The lateral boundary of $\partial \Omega_{\varepsilon}=\partial \Omega\left(\gamma_{\varepsilon}\right)$ is defined by the parametrization

$$
\begin{aligned}
X^{\varepsilon}(u, v)= & \gamma_{\varepsilon}(u)+R \cos v N_{\varepsilon}(u)+R \sin v B_{\varepsilon}(u) \\
= & X(u, v)+\varepsilon\left\{h(u)+R \cos v\left[\frac{h^{\prime \prime}(u)}{\kappa(u)}-\frac{\gamma^{\prime \prime}(u) \cdot h^{\prime \prime}(u)}{\left|\gamma^{\prime \prime}(u)\right|^{2}} N(u)\right]\right. \\
& \left.\quad+R \sin v\left[-\frac{\gamma^{\prime \prime}(u) \cdot h^{\prime \prime}(u)}{\kappa^{2}(u)} B(u)+h^{\prime}(u) \times N(u)+\frac{1}{\kappa(u)} T(u) \times h^{\prime \prime}(u)\right]\right\}+o(\varepsilon)
\end{aligned}
$$

in the space of continuous functions $C$, provided $\gamma, \beta \in C^{3}$, here $0 \leq u \leq l, 0 \leq v<2 \pi$.

Therefore, the speed of $\partial \Omega_{\varepsilon}$ with respect to the parameter $\varepsilon$ at $\varepsilon=0$ is given by

$$
\begin{aligned}
V(h)(u, v)= & \left.\frac{\partial X^{\varepsilon}}{\partial \varepsilon}\right|_{\varepsilon=0}(u, v) \\
= & h(u)+R \cos v\left[\frac{h^{\prime \prime}(u)}{\kappa(u)}-\frac{\gamma^{\prime \prime}(u) \cdot h^{\prime \prime}(u)}{\left|\gamma^{\prime \prime}(u)\right|^{2}} N(u)\right] \\
& \quad+R \sin v\left[-\frac{\gamma^{\prime \prime}(u) \cdot h^{\prime \prime}(u)}{\kappa^{2}(u)} B(u)+h^{\prime}(u) \times N(u)+\frac{1}{\kappa(u)} T(u) \times h^{\prime \prime}(u)\right] .
\end{aligned}
$$

\section{Remark A.2}

(1) In order to have $V(u, v)$ to be $C^{1}$, we need $\gamma \in C^{4}$, since $h$ depends on $\gamma^{\prime}$.

(2) If the condition $\left|\gamma^{\prime \prime}(u)\right| \neq 0$ is not satisfied, we cannot use the Frenet formulas and should directly construct the field $V$ from the given parametrization of $\partial \Omega_{\varepsilon}$. 
Unit `e de recherche INRIA Lorraine, Technop^ole de Nancy-Brabois, Campus scientifique, 615 rue du Jardin Botanique, BP 101, 54600 VILLERS LÈS NANCY

Unit'e de recherche INRIA Rennes, Irisa, Campus universitaire de Beaulieu, 35042 RENNES Cedex Unit'e de recherche INRIA Rh`one-Alpes, 655, avenue de l'Europe, 38330 MONTBONNOT ST MARTIN Unit'e de recherche INRIA Rocquencourt, Domaine de Voluceau, Rocquencourt, BP 105, 78153 LE CHESNAY Cedex Unit'e de recherche INRIA Sophia-Antipolis, 2004 route des Lucioles, BP 93, 06902 SOPHIA-ANTIPOLIS Cedex

\section{Éditeur}

INRIA, Domaine de Voluceau, Rocquencourt, BP 105, 78153 LE CHESNAY Cedex (France)

http://www.inria.fr

ISSN 0249-6399 